
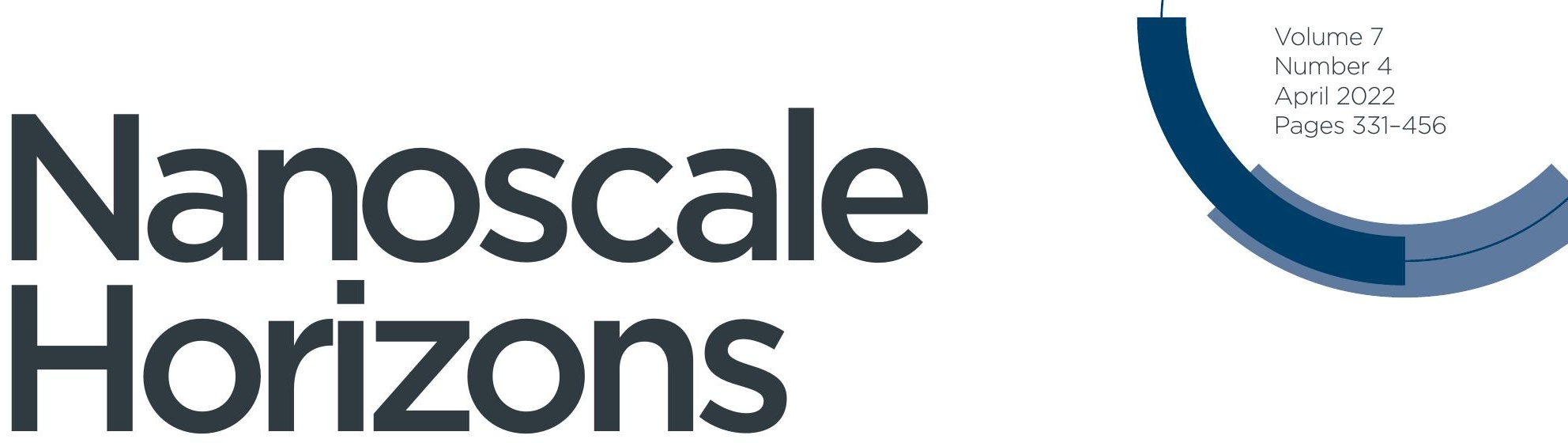

The home for rapid reports of exceptional significance in nanoscience and nanotechnology rsc.li/nanoscale-horizons

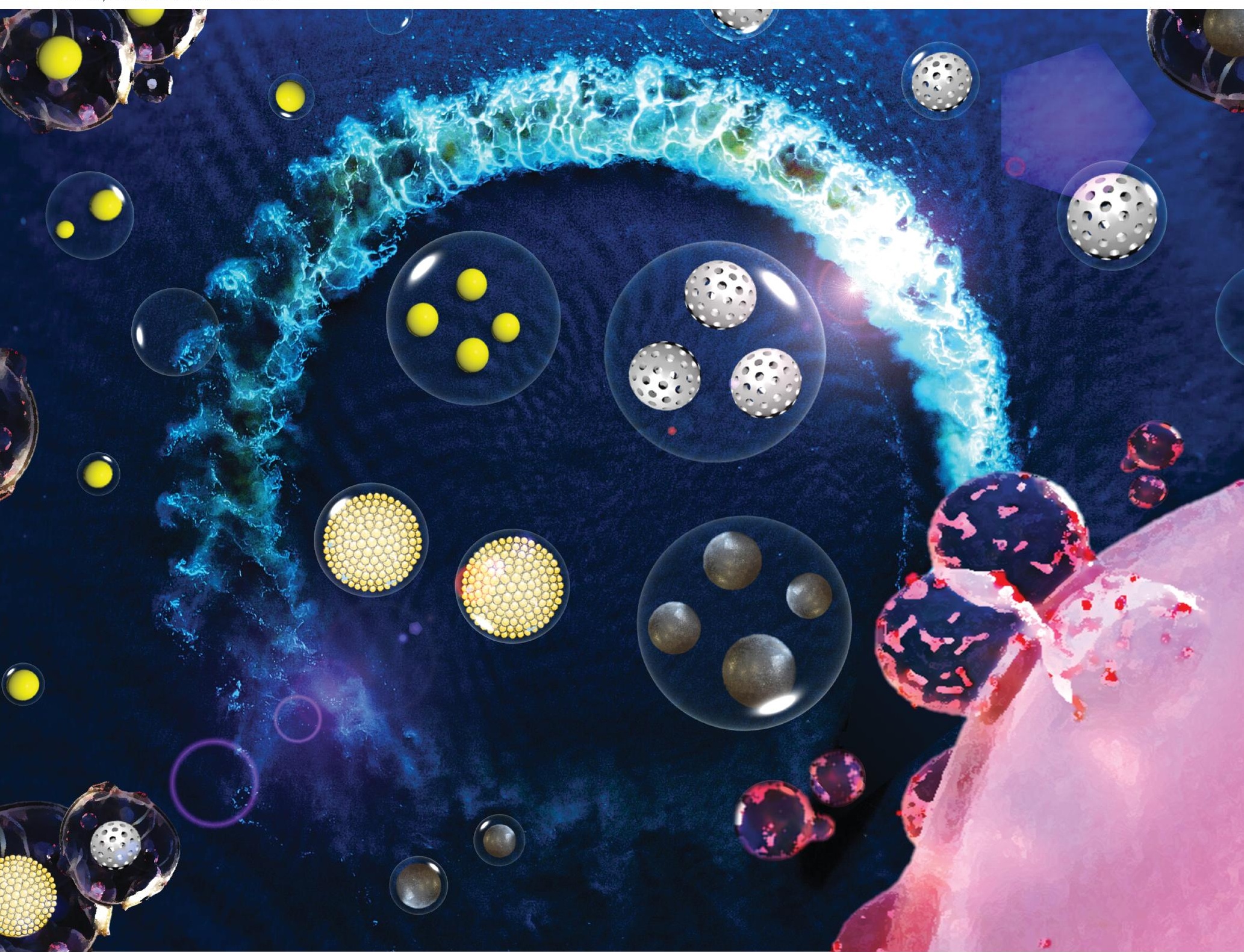

ISSN 2055-6756 
Check for updates

Cite this: Nanoscale Horiz., 2022, 7, 352

Received 6th December 2021. Accepted 11th January 2022

DOI: $10.1039 / \mathrm{d} 1 \mathrm{nh} 00637 \mathrm{a}$

rsc.li/nanoscale-horizons

\section{Integrated therapy platform of exosomal system: hybrid inorganic/organic nanoparticles with exosomes for cancer treatment}

\author{
Ming-Hsien Chan, (D) $\dagger^{\mathrm{a}}$ Zhi-Xuan Chang, $\dagger^{\mathrm{b}}$ Chi-Ying F. Huang, (D) ${ }^{\text {bc }}$ L. James Lee, ${ }^{\text {bd }}$ \\ Ru-Shi Liu (D) *ae and Michael Hsiao (D) *af
}

\begin{abstract}
Recent studies have found that exosomes or extracellular vehicles (EVs) are associated with cancer metastasis, disease progression, diagnosis, and treatment, leading to a rapidly emerging area of exocrine vesicle research. Relying on the superior targeting function and bio-compatibility of exosomes, researchers have been able to deliver drugs to cancer stem cells deep within tumors in mouse models. Despite significant efforts made in this relatively new field of exosome research, progress has been held back by challenges such as inefficient separation methods, difficulties in characterization/tracking, and a lack of specific biomarkers. Therefore, current researches are devoted to combining nanomaterials with exosomes to improve these shortcomings. Adding inorganic/organic nanoparticles such as artificial liposomes and iron oxide can bring more drug options and various fluorescent or magnetic diagnostic possibilities to the exosome system. Moreover, the applications of exosomes need to be further evaluated under actual physiological conditions. This review article highlights the potential of exosomebiomimetic nanoparticles for their use as drug carriers to improve the efficacy of anticancer therapy.
\end{abstract}

${ }^{a}$ Genomics Research Center, Academia Sinica, Taipei, 115, Taiwan. E-mail: mhsiao@gate.sinica.edu.tw

${ }^{b}$ Institute of Biopharmaceutical Sciences, National Yang Ming Chiao Tung University, Taipei, Taiwan

${ }^{c}$ Institute of Clinical Medicine, National Yang Ming Chiao Tung University, Taipei, Taiwan

${ }^{d}$ Department of Chemical and Biomolecular Engineering, Ohio State University, Columbus, 43210, Ohio, USA

${ }^{e}$ Department of Chemistry, National Taiwan University, Taipei 106, Taiwan.E-mail: rsliu@ntu.edu.tw

${ }^{f}$ Department of Biochemistry, College of Medicine, Kaohsiung Medical University, Kaohsiung 807, Taiwan. E-mail: mhsiao@gate.sinica.edu.tw

$\dagger$ Ming-Hsien Chan and Zhi-Xuan Chang contributed equally.

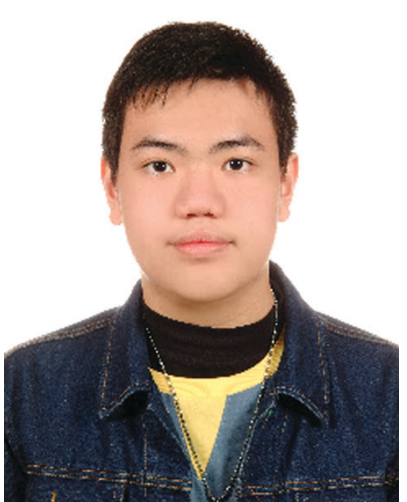

Ming-Hsien Chan
Ming-Hsien Chan received his bachelor's and master's degree in bioscience and biotechnology from National Taiwan Ocean University in 2014. He obtained a PhD degree in chemistry from National Taiwan University in 2018. He is a postdoctoral fellow in Prof. Hsiao's group at Genomics Research Center, and is supported by Academia Sinica Outstanding Post-doctoral Fellowships. His current research interests include the synthesis of biological nanomaterials and their application in vitro and in vivo.

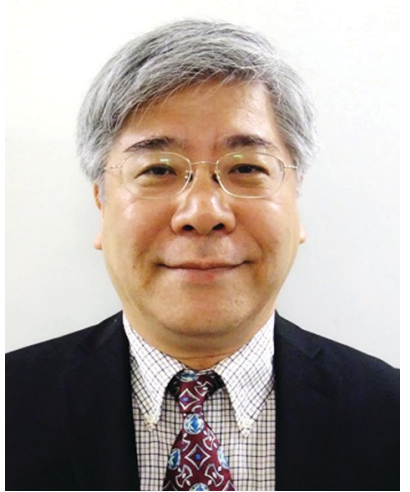

Michael Hsiao
Michael Hsiao is currently a Professor and Research Fellow at the Genomics Research Center, Academia Sinica. He received his D.V.M. degree at National Taiwan University in 1983. He obtained a PhD degree in Pathology from the University of Southern California in 1991. His researches focus on targeting tumor growth/survival pathways to enhance tumor chemosensitivity and/or radiosensitivity, developing novel strategies to overcome tumor metastasis, developing nanoparticlemediated high-throughput transfection platforms, as well as exploring novel cancer theranostics with the next generation nanoparticles. 


\section{Introduction}

Cancer is characterized by the US National Cancer InstituteNational Institutes of Health (NIH NCI) as a significant disease that requires several therapeutic strategies to reduce its current high mortality. Highly aggressive cancer types such as lung adenocarcinoma (LUAD), glioblastoma (GBM), and pancreatic ductal adenocarcinoma (PDAC) originate from organs that are difficult to diagnose and trace. One of the primary reasons these cancers have high mortality rates is the lack of treatment means and diagnosis methods. In an effort to prevent the lifethreatening scenarios caused by these high-fatality cancers, exosomes are considered as a new generation of nano drugs with both therapeutic and diagnostic functions. ${ }^{1-3}$ Many studies have found that exosomes derived from tumor cells contain an abundance of specific microRNAs, and their biochemical properties render them stable and easy to store. ${ }^{4-6}$ When exosomes are designed or selectively encapsulated with unique biologically active molecules, they can be used to deliver tumor suppressor molecules or chemotherapy drugs. ${ }^{7}$ Moreover, exosomes are considered an excellent candidate for GBM treatment since they can be developed as a multifunctional drug system that can penetrate the blood-brain tumor barrier. ${ }^{8}$ Exosomes have many functions and can penetrate physiological barriers in the brain and pancreas, and their therapeutic efficacy has been successfully demonstrated in previous research works. ${ }^{9}$

Currently, many researchers are developing new technologies that can produce therapeutic exosomes (tExos) containing multiple therapeutics and targeting ligands as a new drug platform to treat cancers. $^{10}$ Although exosomes have many advantages, many challenges remain that need to be overcome for their use in disease treatment and diagnosis. These problems can be solved by combining exosomes with inorganic/organic nanomaterials to optimize the medical application value of exosomes. The following are several essential issues that such hybrid nanomaterials can address. (1) The total number of exosomes consumed in the treatment is too high. Based on current research, exosomes carrying genes or proteins require a dose of $>10^{10}$ particles per $\mathrm{mL}$ to achieve a therapeutic effect. On the other hand, when combined with inorganic/organic nanomaterials, only $10 \mathrm{ng} \mathrm{mL} \mathrm{m}^{-1}$ of the drug is necessary to produce the same therapeutic effect. ${ }^{11}$ In detail, compared with other nanomaterials, the drug-carrying capacity of exosomes is challenging o control. The drugs needed to be sent into the cell first, then collected and purified the exosome with the drug in the cell supernatant.

However, inorganic/organic nanomaterials can preferentially carry and pack medicines with known concentrations in bench synthesis, protecting the drug molecules that won't react with the unpredictable cellular environment, such as protein or nucleic acid substance and decreasing the drug dose. (2) Exosomes in circulation are quickly taken up by immune cells in the body and are difficult to detect. ${ }^{12}$ After modification with specific ligands, inorganic/organic nanomaterials can prevent the exosome uptake by immune cells. Moreover, these particles can be detected using luminescence, ultrasonic signaling, and magnetic properties for diagnosis. (3) The central core of the exosome is hydrophilic. As a result, exosomes cannot load hydrophobic drugs quickly. Since the center of some inorganic/organic nanomaterials has hydrophobic pores or spaces, they thereby allow the particles to carry hydrophobic drugs, which can then be enveloped with exosomes for delivery to cancer cells. ${ }^{13,14}$ Inorganic/organic nanoparticles hybridized with exosomes have unique properties, such as enhanced permeability and retention (EPR) effect, modifiability, intelligent responsiveness, and co-delivery of multiple drugs. These materials play an essential role in the precise and synergistic treatment of tumors by enhancing efficacy and reducing toxicity and side effects. Moreover, hybrid nanomaterials are often coupled with targeting molecules to achieve the targeted delivery of antitumor drugs. ${ }^{15}$ These biomimetic nanoplatforms combine the unique functions of natural biomaterials and the versatility of artificial nanomaterials, resulting in anti-tumor nanomedicines with five essential characteristics: long circulation time, tumor targeting, deep tumor penetration, high cellular internalization, and strong drug release (Fig. 1). In this review, we first introduce the properties of exosomes, followed by the description of the cancer applications of exosomes with a deep focus on how exosomes are combined with inorganic/organic nanomaterials to provide multiple and beneficial therapeutic and diagnostic results.

\section{Exosomes}

\subsection{The background of exosomes}

Cell-secreted extracellular vehicles (EVs), particularly exosomes with sizes of $30-150 \mathrm{~nm}$ in diameter, encapsulating various active biomolecules, including nucleic acids, lipids, and proteins, have emerged as promising therapeutic agents. Unpurified exosomes belong to a larger group of cell-derived heterogeneous vesicles defined as EVs produced and released by cells.

EVs are distinct in size and biogenesis, and there are three main types of double-layer vesicles: exosomes, apoptotic bodies, and microvesicles. Apoptotic bodies are $0.5-5 \mu \mathrm{m}$ in diameter; they are produced and released by dying cells that undergo apoptosis. Microvesicles are 50-1000 $\mathrm{nm}$ in diameter and are formed by "pinching off" from the plasma membrane. Exosomes are 30-200 $\mathrm{nm}$ in diameter. They are intraluminal vesicles (ILVs) in multivesicular bodies (MVBs), which are organelles involved in the intracellular traffic of biomolecules and released via inward budding at the plasma membrane (Fig. 2). ${ }^{16}$ Exosomes were first reported 30 years ago. In the 1980s, exosomes were discovered by researchers working with maturing reticulocytes. ${ }^{17}$ They observed that small vesicles created by inward budding in the intracellular endosome would form a multivesicular body (MVB). The MVB would then fuse with the cell plasma membrane (PM). The internal vesicles would be secreted from cells as exosomes. ${ }^{18,19}$ In the past, exosomes were considered to be the dustbins of cells, but due to recent discoveries of their desirable features for use in cell communication, they are expected to become a new type of biomarker for disease diagnosis and drug nanocarriers. In addition to their biocompatibility (due to their presence in 


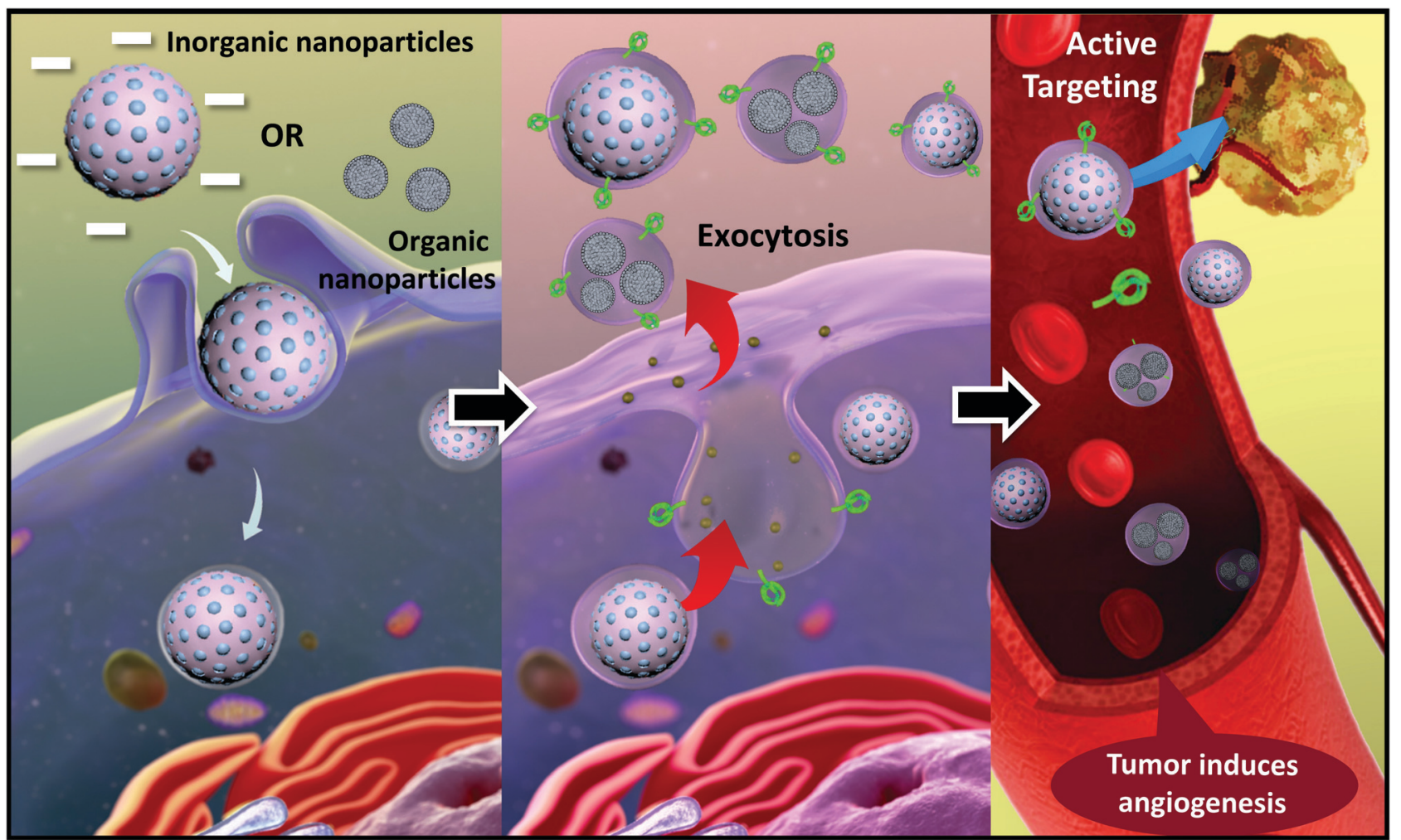

Fig. 1 Exocytosis refers to the process in which nanomaterials in cells fuse with the plasma membrane through vesicles and are released into the extracellular matrix with the help of the fusion protein. These nanomaterials are released into the extracellular matrix using the fusion protein. The process can be regarded as the reverse effect of cellular endocytosis. Exocytosis can occur spontaneously or be triggered by other signals. After encapsulating an exosome membrane, the hybrid particles can track the tumor via specific surface proteins.

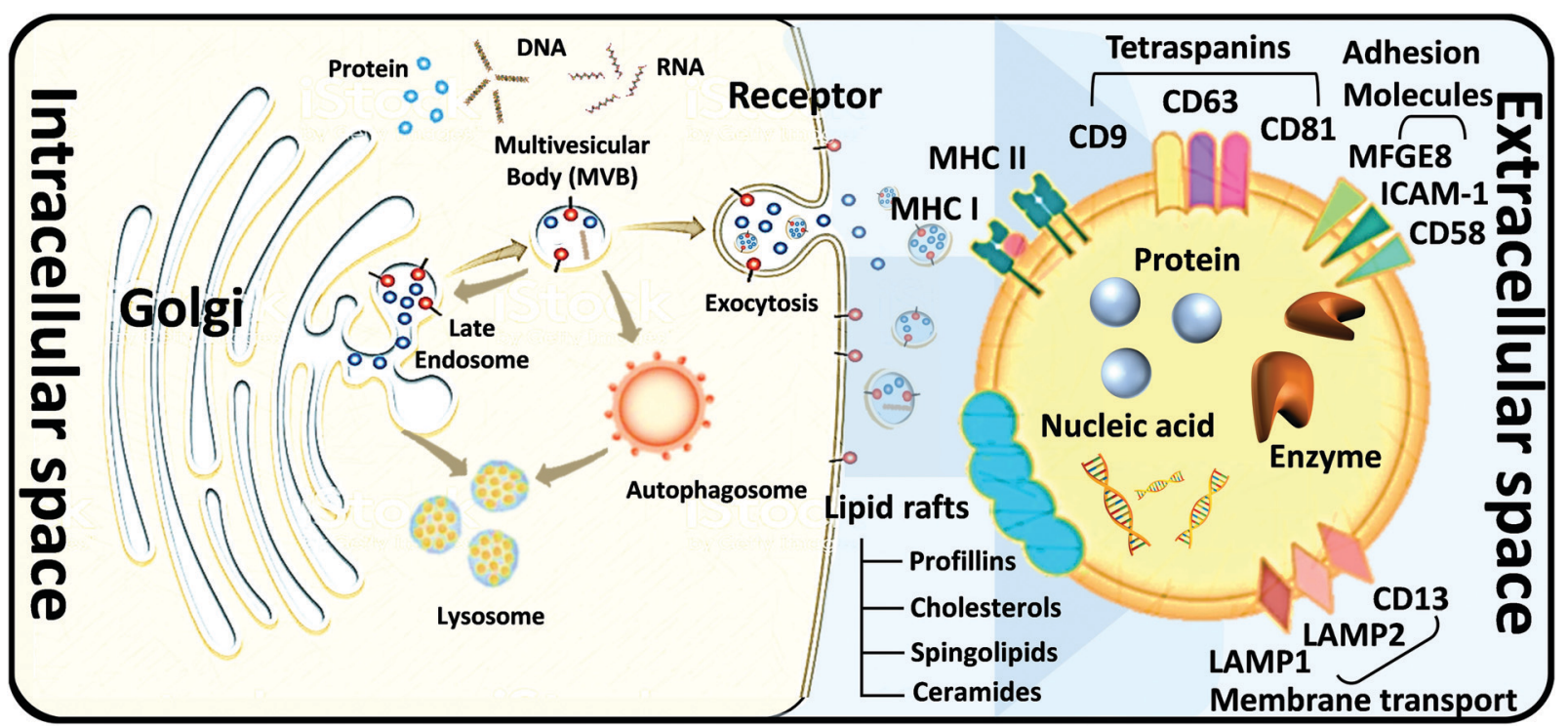

Fig. 2 Biogenesis and application of exosomes. Biogenesis of exosomes. Three routes of exosomal biogenesis. Exosome composition, including (1) MFGE8: milk fat globule-EGF factor 8 proteins; (2) ICAM-1: intercellular adhesion molecule 1; (3) LAMP1,2: lysosomal-associated membrane protein 1, 2; (4) MHC I, II: major histocompatibility complexes I, II; (4) MAPK: mitogen-activated protein kinase; ERK: extracellular signal-regulated kinase; (5) GAPDH: glyceraldehyde 3-phosphate dehydrogenase; PGK1: phosphoglycerate kinase 1.

many body fluids and tissues), exosomes have intrinsic transmembrane and membrane-anchored proteins that provide prolonged blood circulation and promote tissue-directed delivery and cellular uptake of their internal contents. Exosomes are 
usually formed in three steps: (1) endocytosis of extracellular material, (2) maturation, and (3) exocytosis.

\subsection{Isolation, purification, and analysis of exosomes}

How to obtain and confirm high-purity exosomes is currently a hot research topic. Most of the current methods of combining nanoparticles with exosomes as a composite nano-platform are first to use cells as providers of exosomes, then add target nanomaterials to the cells for cell uptake, and finally release the package through exocytosis. After these procedures, a composite carrier covered with exosomes and target nanomaterials was born. Therefore, isolation, purification, and analysis of exosomes are also important topics. Here is a brief description of several popular ways to connect the hybrid inorganic/organic nanoparticles with exosomes. High-speed UC, which is regarded as the most common isolation method, mainly depends on vesicle size and density. Gradient-based UC can also be applied further to purify the exosomes to a narrower distributed population. ${ }^{20}$ Ultrafiltration membranes, including tangential flow filtration (TFF), can also be used to isolate exosomes based on vesicle sizes. ${ }^{21}$ Depending on the size of the membrane, this method allows for the separation of exosomes from proteins and other macromolecules. Immunoaffinity methods utilize interactions between antibodies and selective exosome surface proteins to isolate the exosomes or exosome subpopulations. ${ }^{21}$ Size exclusion chromatography (SEC) relies on resin-filled porous columns to sort EVs and exosomes into fractions based on their different retention times in the column. ${ }^{20}$ In the precipitation method, exosomes can be isolated from biological fluids by changing their solubility. ${ }^{22}$

Other techniques apply polymers to precipitate exosomes based on their surface features and polymer molecular weights. ${ }^{23}$ After exosomes are sorted and collected from a sample, and they are characterized by size, protein expression, RNA content, and lipid composition. Several techniques can be applied, such as nanoparticle tracking analysis (NTA), dynamic light scattering (DLS) and nanoscale flow cytometry for exosome size and number quantification, LC-MS, western blot, and ELISA for protein profiling and characterization, Next Generation Sequencing (NGS), microarray, and qPCR for RNA profiling and characterization, and several microscopy techniques for exosome morphology characterization. ${ }^{24-26}$ In the following, we briefly describe several widely used methods for exosome analysis. Nanoparticle Tracking Analyzer (NTA) uses laser light to irradiate a nanoparticle suspension. The particle size, scattered light intensity, quantity, concentration, and dynamic images of moving particles under Brownian motion can be obtained at the same time (Fig. 3a). ${ }^{27,28}$ The working principle of the Transmission/ Scanning Electron Microscope (TEM/SEM) is the use of an electron beam with an energy of 1-30 kV to irradiate the surface of the analyzed sample in the raster scan mode. ${ }^{29}$ Secondary electrons and backscattered electrons are imaged to obtain information on the microstructure and morphology of the sample surface and have high resolution (Fig. 3b). Dynamic light scattering (DLS) is a technique that collects fluctuations in the intensity of the scattered light of particles undergoing Brownian motion in the solution. ${ }^{30}$ These light intensity fluctuations are converted into a correlation curve through an appropriate instrument, thereby obtaining their speed (Fig. 3c).

In addition, in 1989, Sidney Altman and Thomas Cech discovered that small RNA or DNA has functions such as ligand binding, activation, and gene regulation. They can be screened for secondary structure RNA or single-stranded DNA that specifically binds to the target protein. These specialized small molecule nucleic acids are called aptamers. Aptamers can also be used for exosome detection, such as the fluorescence method for exosome competitive detection of CD63-specific aptamers (Fig. 3d). In the presence of exosomes, CD63 on the exosomes binds to the aptamer, resulting in the shedding of short sequences into the supernatant. The number of exosomes can be estimated by detecting the fluorescence intensity in the supernatant. Compared with conventional methods, the detection cost and difficulty are significantly reduced, while the sensitivity is guaranteed, which provides a basis for subsequent detection of specific cancer cell exosomes.

These standard analysis methods have their advantages and drawbacks. Therefore, evaluating exosomes from multiple perspectives is necessary, especially for hybrid exosomes where inorganic/organic nanoparticles may lead to significant exosome size and morphology changes.

\section{The cancer applications of exosomes and hybrid with inorganic/ organic nanoparticles}

At first, exosomes were considered excess membrane proteins released by the regulating membrane function during cell maturation. They were characterized as organelles that remove cell debris and eliminate cell surface molecules. Later, an increasing number of studies found that exosomes play essential roles in mediating inflammation and cell proliferation, regulating the extracellular microenvironment, and inducing the body's immune response. ${ }^{32}$ At present, exosomes are considered biomarkers and prognostic factors of diseases, and they have the potential to serve as carriers for gene and drug delivery, which have important clinical significance. ${ }^{33-35}$

\subsection{Diagnostic and prognostic markers of cancer}

Because exosomes can be detected in body fluids (blood, urine, saliva, and cerebrospinal fluid), they are considered noninvasive or minimally invasive biomarkers for disease diagnosis. They can detect many pathological conditions, including cancer potential (Fig. 4a). ${ }^{36-39}$ The current focus of genomic medicine is to develop tests based on personalized and precision medicine. Therefore, a liquid biopsy that uses body fluids (mainly blood and urine) instead of tissue samples obtained through surgery for diagnosis is rapidly attracting attention. This method is convenient for patients with minimal harm. The liquid biopsy covers three significant types, including circulating tumor cells (CTCs), free DNA (cfDNA), and exosomes (or EVs). Exosomes are the most attractive type in liquid biopsy due to their high 
(a)

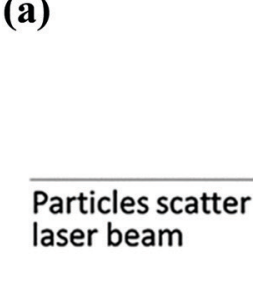

\section{Particles to be viewed are suspended in liquid}

Laser beam

(Approximately $50 \mu \mathrm{m}$ wide)

(c) (b)

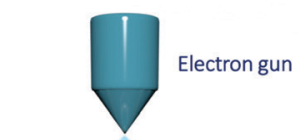

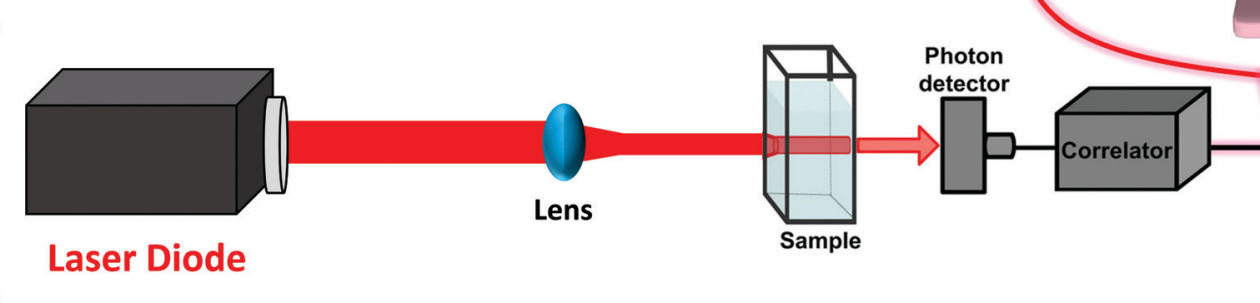

(d)

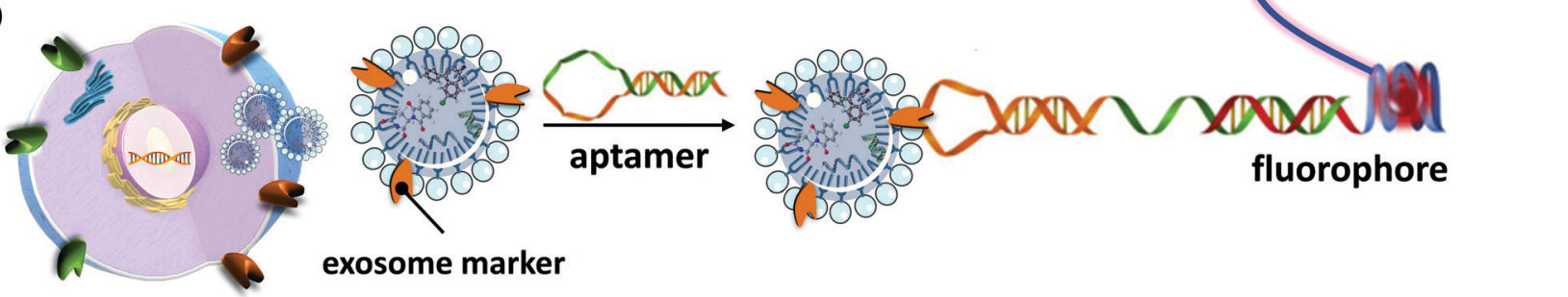

Fig. 3 Different methods of analyzing exosomes. (a) Nanoparticle Tracking Analysis (NTA) has become essential for exosome research. NTA technology provides dynamic visual images, (b) scanning Electron Microscope (SEM) offers morphological identification of exosomes. (c) Dynamic light scattering (DLS) is a physical characterization method used to measure the particle size distribution in a solution or suspension. (d) Employing a specific aptamer for exosome detection with a fluorophore.

(a)

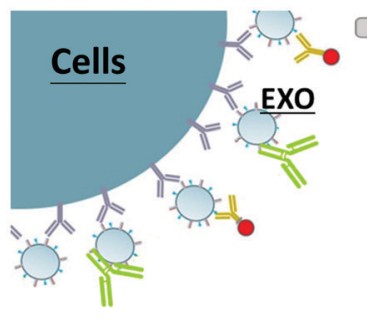

(b)
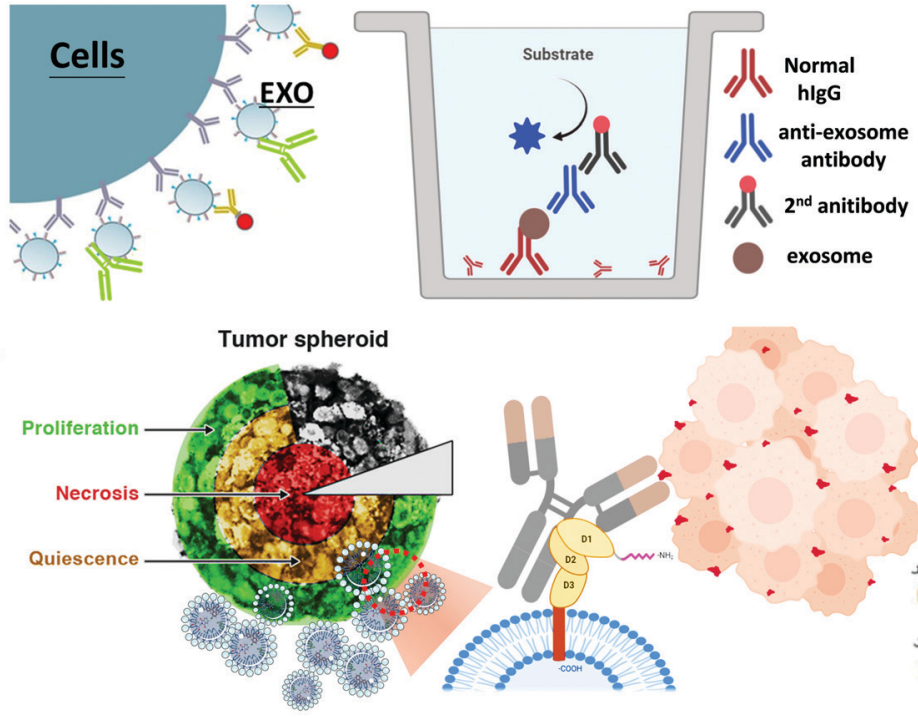

(c)

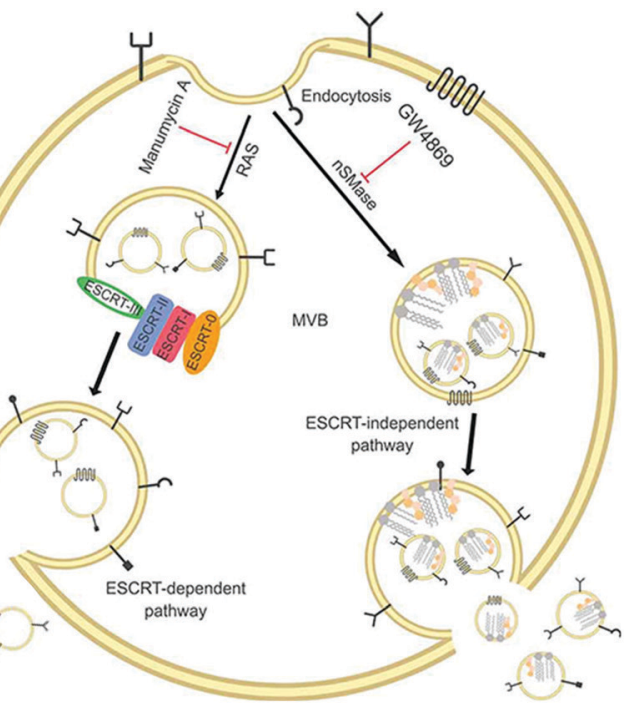

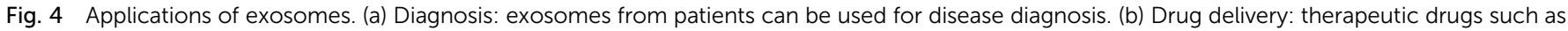

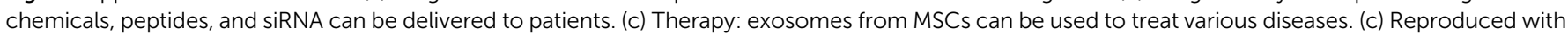
permission. ${ }^{31}$ Copyright 2020, Wiley Online Library. 
stability and ease of collection from body fluids such as urine and blood. miRNAs are considered potential diagnostic biomarkers because they play a regulatory role in the body. Changes in exosomal miRNA enrichment can reflect physiological and pathological changes, indicating their possible use as diagnostic biomarkers. According to reports, miR-23b-3p, miR-10b-5p, and miR-21-5p are promising prognostic biomarkers for non-small cell lung cancer (NSCLC) patients. ${ }^{40-42}$ In addition to miRNA, exosomes also contain proteins from host cells, so they have potential functions as biomarkers. Analysis of the entire proteome of exosomes by mass spectrometry has highlighted various biomarkers in prostate and bladder cancer. ${ }^{43}$ A study involving 276 NSCLC patients identified NY-ESO-1, EGFR, PLAP, EPCAM, and Alix in exosomes as protein biomarkers for overall survival. ${ }^{44}$ Plasma-derived exosomes have been successfully used to evaluate multi-label models using the EV Array system, consisting of 37 antibodies against lung cancer-related proteins. ${ }^{45}$ This method uses only $10 \mu \mathrm{L}$ of plasma samples for analysis. Exosomal lncRNA is also defined as a new tumor biomarker. ${ }^{46}$

\subsection{Drug delivery vehicles}

One of the most promising drug delivery systems is liposomes. Liposomes are artificial vesicles with a phospholipid membrane self-assembled into different sizes and forms in an aqueous milieu. Liposomes allow for long circulation and a wide range of techniques for high concentration drug loading, and they can be prepared easily. However, the immunocompatibility of liposomes is debatable, and their targeting capability in vivo is often insufficient. ${ }^{47}$ Compared to liposomes, exosomes are more stable in circulation because of their endogenous nature and unique surface content. ${ }^{48}$ Due to their small size and bubble shape, exosomes can move from one cell to another, release their contents through the cell membrane. Due to their protein content and genetic material, biological and small drugs can be incorporated into exosomes. An example of such a system is doxorubicin-loaded exosomes. ${ }^{49}$ Once exosomes have been internalized into recipient cells, and their cargo can exert its effects. In a 2007 study, the authors prove that exosome mRNA can be translated into recipient cells. ${ }^{50}$ They first collected exosomes from mice and then incubated them with human cells. After incubation, three different mouse proteins were found in human recipient cells, indicating that exosome cargos can be transferred into cells. ${ }^{51}$ Exosome-based therapy has several significant advantages: (1) low immunogenicity; (2) no vascular occlusion; and (3) systemic injection of exosomes can cross the blood-brain barrier and enter the brain parenchyma. ${ }^{52}$

Recently, post-insertion of siRNA into exosomes by conventional bulk electroporation has been developed and demonstrated success; the performance was superior to that of synthetic nanocarriers in several mouse models for central neuron and cancer treatment. ${ }^{53-55}$ Many clinical trials on exosome therapy have also been launched worldwide. ${ }^{56}$ Despite their many advantages, the application of exosomes in gene delivery for clinical use has been slow because producing sufficient quantities for large-scale in vivo use is technically challenging. Even though the exosome dose $\left(\sim 50 \mu \mathrm{g} \mathrm{kg}^{-1}\right.$ or $\sim 1 \mu \mathrm{g}$ exosomal RNA from $\sim 10^{9}$ exosomes in one injection per mouse, similar to an adeno-associated virus dose) is lower than using synthetic RNA and nanocarriers $\left(\sim 1 \mathrm{mg} \mathrm{kg}^{-1}\right)$, large numbers of cell cultures are required. It takes days of incubation, followed by purification and nuclei acid loading, to generate gene-containing exosomes in sufficient quantities for repeated use in human therapy because 150-200 times more exosomes are needed for a patient than for a mouse. Only a few cell types, such as mesenchymal stem cells (MSCs) and dendritic cells (DCs), are known to secrete relatively high numbers of exosomes, e.g., $>5 \times$ $10^{4}$ per cell in 1-2 days, but the strong electric field required to insert RNAs would damage many exosomes, leading to a low yield. Post-insertion of large biomolecules into nano-sized exosomes is also inefficient. New technologies to generate many exosomes with a high loading of RNAs and targeting peptides in exosomes are essential for nucleic acid-based exosome therapy.

\subsection{Anticancer vaccine for cancer immunotherapy}

Recently, the success of immune checkpoint therapy in several cancer types has increased people's interest in the further exploration of immune disorders in tumors, including exosomal tumor immunotherapy, which is expected to produce anticancer vaccines (Fig. 4b). ${ }^{57}$ Tumor-derived exosomes carry antigens and have been used as a source of specific stimulation of the immune response against tumors. One study found that B cells transformed with Epstein-Barr virus released exosomes containing major histocompatibility complex (MHC)-II, thereby activating CD4+ T cells and the exosomes secreted by dendritic revealed to express MHC-I, which activates CD8+ T cells. ${ }^{58}$ Subsequent studies found that those exosomes can induce anti-tumor immunity in experimental animal models and human clinical trials, including colorectal cancer, metastatic skin cancer, and non-small cell lung cancer. ${ }^{59-61}$ In mice, tumor-derived exosomes can serve as an antigen delivery system and prevent the development of autologous tumors in a CD4+ and CD8+ T cell-dependent manner. ${ }^{62}$ In phase I clinical trial, patients with advanced non-small-cell lung cancer or metastatic melanoma were vaccinated with dendritic cell-derived exosomes and showed an anti-tumor immune response and tumor regression. Recent evidence showed that dendritic cells loaded with tumor exosomes effectively produce an anti-tumor response against autologous tumor cells in patients with malignant glioma. ${ }^{63}$

Researchers believe ascites-derived exosomes are as effective as dendritic cells or tumor-derived exosomes in sensitizing dendritic cells and the primary cytotoxic $\mathrm{T}$ lymphocytes, thereby killing autologous tumor cells in vitro. ${ }^{64}$ Moreover, exosomes derived from the malignant effusion of ovarian cancer patients have been prepared and will undergo clinical trials. ${ }^{65}$ It was found that the combination of ascites-derived exosomes from colorectal cancer patients and granulocytemacrophage colony-stimulating factors can effectively induce effective carcinoembryonic antigen-specific anti-tumor immunity in patients with advanced colorectal cancer. ${ }^{66}$

\subsection{Inhibition of exosomes}

Elevated levels of exosomes are usually associated with the progression of different types of cancer. Some researchers hope 
to prevent poor patient prognosis by reducing tumor exosomes to normal levels (Fig. 4c). ${ }^{31,67}$ From this perspective, many ongoing studies aim to regulate the release of exosomes by modulating the process of exosome secretion or by explicitly targeting their components to inhibit their interaction with target cells. Currently, our understanding of exosomal mechanisms and functions under different physiological and pathological conditions is increasing. Although its biological functions have not yet been fully resolved, researchers have conducted in-depth explorations in many fields. Exosomes are not waste particles but a key medium for cell-to-cell communication. As the satellites of host cells, exosomes contain a large amount of biological information, and their functions exceed initial expectations. Host cells control the contents of exosomes, thus changing their fate or that of other cells. Secondly, exosomes have a strong influence on tumor progression and metastasis, and they can be used to predict the location of metastasis and to establish a pre-metastasis niche. Additionally, the exosomes secreted by tumor cells can either block tumor growth by promoting the anti-tumor immune response or induce tumor growth by weakening anti-tumor immunity or promoting angiogenesis. ${ }^{6}$

In the late stage, cancer cell-derived exosomes produce much stronger immunosuppressive effects than immune activation, and vaccines based on antigen-carrying exosomes are also in clinical trials. ${ }^{69}$

Although the use of exosomes for diagnosis and treatment is still in its infancy, their use as a carrier combined with gene therapy has excellent clinical application potential with the deeper understanding and systematic evaluation of exosomes.

\subsection{Advantages of exosomes-inorganic/organic nanoparticles hybrid system}

Organic/inorganic nanocomposites are usually organic polymer composites with inorganic nanoscale structural units. They combine the advantages of inorganic materials (such as rigidity, thermal stability) and organic polymers (such as flexibility, dielectric properties, elasticity, and processability). Exosomes themselves is multifunctional organic carrier, and their lipid bilayer structure is like liposomes. It has excellent compatibility in a biological environment. Combining inorganic materials can significantly improve the biocompatibility of inorganic materials and reduce the release of metal ions generated when inorganic materials are degraded into the biological environment to cause physical toxicity. In addition, the inherent characteristics of inorganic materials, such as fluorescent development, magnetic tracking, etc., also provide exosomes with tracking and monitoring effects. In addition to combining with inorganic nanomaterials, Exosomes can also be easily integrated with organic nanoparticles such as liposomes for membrane fusion. Liposomes are preparations formed by encapsulating drug molecules in vesicles formed by phospholipid bilayer membranes. Since the basic structure of the biological plasma membrane is also a phospholipid bilayer membrane, liposomes have a design similar to exosomes. They both have good biocompatibility that can be easy for the bioapplications. By incorporating targeted substances or chemotherapeutic drugs into the liposome membrane, this artificial organic nanoparticle can deliver the cargos in the cavity to the exosome. ${ }^{87}$ The modified exosome can also carry medicines and post-modified targeted substances.

Mixed functional materials composed of inorganic/organic ingredients are considered a potential platform for expanding the diversified fields of medicine. Combining exosome and organic/organic nanoparticles can produce innovative materials by modifying the composition on the molecular scale. And balance the advantages between the two to adjust the size, design, function, and form. The following chapters are the critical part of this manuscript. We will describe and discuss the contributions of various exosome hybrid organic-inorganic composite materials in the past five years.

\section{Hybrid inorganic/organic nanoparticles with exosomes}

Due to their unique properties, hybrid nanomaterials have recently been studied as promising platforms for imaging and therapeutic applications (Table 1). Since the hybridization of inorganic/organic nanoparticles with exosomes is still a relatively novel technology, many novel types of research have been developed in 2021. This table has sorted out the relevant literature published in the past five years and selected several representative works for in-depth review. Nanotechnology offers great potential for future biomedical applications. ${ }^{88}$ Such nanoparticles can retain the valuable features of their inorganic and organic parts and systematically modify the properties of hybrid exosomes through the combination of functional elements. Furthermore, the conjugation of targeting moieties on the surface of these nanomaterials or exosomes provides a nanoplatform with specifically targeted imaging and therapeutic properties. This section summarizes the latest studies on synthesizing inorganic/organic nanoparticles with exosomes and their applications in the biomedical field. This paragraph integrates some relevant references that point out those specific nanoparticles can be used to optimize the functions of exosomes and improve their shortcomings to obtain a more complete clinically applicable nanotherapeutic theranostic platform.

\subsection{Exosome-liposome hybrid nanoparticles}

Liposomes are formed by encapsulating drug molecules in vesicles formed by a phospholipid bilayer membrane. ${ }^{89}$ Since the basic structure of the biological plasma membrane is also a phospholipid bilayer membrane, and liposomes have a structure similar to biological cells, so they have good biocompatibility (Fig. 5a). ${ }^{90}$ After entering the human body, liposomes act as an "invader" to activate the immune mechanism. They are swallowed by the reticuloendothelial system, thereby causing targeted enrichment in tissues such as the liver, spleen, lung, and bone marrow. This phenomenon is known as the passive targeting of liposomes. Certain targeting substances can be incorporated into the liposome membrane; in this way, liposomes can be targeted to a specific site through biological or physical factors. 
Table 1 The list of hybrid inorganic/organic nanoparticles with exosomes for biomedical applications

\begin{tabular}{|c|c|c|c|c|c|}
\hline \multicolumn{2}{|c|}{ Combination nanoparticles } & $\begin{array}{l}\text { Biological target (source of } \\
\text { exosomes) }\end{array}$ & Theranostic methods & Cancer type & Ref. \\
\hline \multirow{10}{*}{\multicolumn{2}{|c|}{ Liposome }} & Exosome (HEK293T) & RT-PCR & Mesenchymal stem cell & 2018, Lin et al..$^{70}$ \\
\hline & & & Runx2 expression (cell) & & \\
\hline & & Exosome (Fibroblasts) & NBD (Liposome) & Metastatic peritoneal & 2020, Lv et l. $^{71}$ \\
\hline & & CD47-SIRPa & $\begin{array}{l}\text { CD9 antibody } \\
\text { (Exosome) } \\
\text { DIR (gETL) }\end{array}$ & cancer & \\
\hline & & Exosome (HT29) & DOX & Colorectal cancer & 2021, Kwon et al..$^{72}$ \\
\hline & & Exosome (CT26) & CD9 & Colorectal cancer & 2021, L. Cheng et al. ${ }^{73}$ \\
\hline & & CD47-SIRPa & Photothermal therapy & & \\
\hline & & ICG/R837 & & & \\
\hline & & $\begin{array}{l}\text { Exosome (Fibroblast) } \\
\text { Clodronate }\end{array}$ & CD9, Calcein & Pulmonary fibrosis & 2021, L. Sun et al. ${ }^{74}$ \\
\hline & & $\begin{array}{l}\text { CXCR4, miRNA, Antagomir-188 } \\
\text { Exosome (NIH-3T3) }\end{array}$ & CXCR4, CD9 & Osteolytic cancer & 2021, Y. Hu et al. ${ }^{75}$ \\
\hline \multirow[t]{2}{*}{$\begin{array}{l}\text { Metal-organic } \\
\text { framework (MOF) }\end{array}$} & $\mathrm{Zn}$ & Exosome (MDA-MB-231) & $\begin{array}{l}\text { FluoBSA } \\
\text { pH change (cell) }\end{array}$ & Breast cancer & 2018, Cheng et al. ${ }^{76}$ \\
\hline & $\mathrm{Fe}$ & $\begin{array}{l}\text { Exosome (HeLa) } \\
\text { Folate }\end{array}$ & Calcein & Cervical cancer & 2017 , Illes et al. ${ }^{77}$ \\
\hline \multirow[t]{10}{*}{$\begin{array}{l}\text { Inorganic } \\
\text { nanoparticles }\end{array}$} & Porous silicon & Exosome (H22 or Bel7402) & DOX & $\begin{array}{l}\text { Liver cancer } \\
\text { Lung cancer }\end{array}$ & 2019 , Yong et $a .^{78}$ \\
\hline & & CD54 & & $\begin{array}{l}\text { Breast cancer } \\
\text { Melanoma }\end{array}$ & \\
\hline & $\begin{array}{l}\text { Quantum dots } \\
\text { (black } \\
\text { phosphorus) }\end{array}$ & $\begin{array}{l}\text { Exosome (LLC cells or Hepa1-6) } \\
\text { tumor antigen }\end{array}$ & DIR & $\begin{array}{l}\text { Lung cancer } \\
\text { Liver cancer }\end{array}$ & 2020, Liu et al. ${ }^{79}$ \\
\hline & $\mathrm{MnCO}$ & Exosome (4T1) & $\begin{array}{l}\text { CD63 antibody } \\
\text { CD9 antibody }\end{array}$ & Breast cancer & 2021, Zhu et al. ${ }^{80}$ \\
\hline & $\mathrm{Fe}_{3} \mathrm{O}_{4}$ & $\begin{array}{l}\text { Exosome (Rat) } \\
\text { MLC and CD63 antibody }\end{array}$ & $\begin{array}{l}\mathrm{RhB} \\
\mathrm{PKH} 26\end{array}$ & Myocardial infarction & 2020, Liu et al. ${ }^{81}$ \\
\hline & & Urinary exosome & UV-Vis & Prostate cancer & 2021, Pan et al. ${ }^{82}$ \\
\hline & Gold (Au) & $\begin{array}{l}\text { Exosome (HEK293T) } \\
\text { RVG Lamp2b }\end{array}$ & $\begin{array}{l}\text { DOX } \\
\text { Dil }\end{array}$ & Brain cancer & 2019, Khongkow et al. ${ }^{83}$ \\
\hline & & Exosome (MSC) & CD63 antibody & Mesenchymal stem cell & 2019, Sancho-Albero et al. ${ }^{84}$ \\
\hline & & Exosome (B16-F10) & PKH67 & Melanoma & 2019, Sancho-Albero et al. ${ }^{85}$ \\
\hline & & Exosome (FBS) & $\begin{array}{l}\text { Temperature (cell) } \\
\text { pH change (cell) }\end{array}$ & $\begin{array}{l}\text { Colon cancer } \\
\text { Breast cancer } \\
\text { Cervical cancer }\end{array}$ & 2021 , Lee et $a .^{86}$ \\
\hline
\end{tabular}

The adding ligands provide the active targeting of liposomes, and it has emerged as an essential mechanism in drug delivery.

Techniques include heat-sensitive liposomes, magnetically guided liposomes, and antibody-guided liposomes (Fig. 5b and c). ${ }^{91-93}$ However, at present, several limitations remain. First, its preparation technology introduces specific difficulties to industrial production; additionally, the encapsulation rate is low for some water-insoluble drugs, which easily leak from liposomes. Poor stability is also a problem in the commercialization of liposomes. The current freeze-drying method may be an effective way to extend the storage period of liposomes. Identification by the immune system is also a challenge encountered by liposomes. The exosome itself can evade immune identification, combined with the active targeting of liposomes to create a new drug hybrid carrier system. Exosomes are a promising drug delivery vehicle. However, it is challenging to encapsulate large nucleic acids in exosomes due to their small sizes. The current reports on exosomes as drug delivery vehicles are mainly related to small nucleic acids such as miRNA, siRNA, or low-molecular-weight drugs, which are much smaller than large plasmids, such as those expressing Cas9, with a minimum size of 5-6 kb. Therefore, there is an urgent need to find a suitable method to encapsulate the CRISPR/Cas9 system into exosomes. Lin et al. successfully encapsulated large nucleic acids, including CRISPR/Cas9 expression vectors, into hybrid exosomes produced by incubating primitive exosomes with liposomes (Fig. 5d and e). ${ }^{70}$ The loaded hybrid exosomes can be endocytosed by mesenchymal stem cells (MSCs) and release cargo.

The hybrid exosome delivery CRISPR/Cas9 system has been verified to be capable of expression in MSCs and regulate the expression of target genes. These target genes provide a new strategy for delivering the CRISPR-Cas9 system to MSCs for gene manipulation. Further research has shown that liposomes can offer additional advantages to exosomes. To improve the therapeutic effect of exosome-liposome hybrid nanoparticles, hyperthermic intraperitoneal chemotherapy (HIPEC) was recently developed. Lv et al. synthesized a genetically engineered exosome-thermosensitive liposome hybrid (gETL). They loaded it with docetaxel (DTX) to analyze its characteristics in cell line-derived (CDX) and patient-derived tumor xenografts (PDXs) for MPC treatment. ${ }^{87}$ They found that when gETL was 
(a)

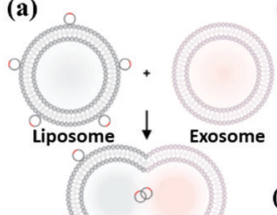

(b)

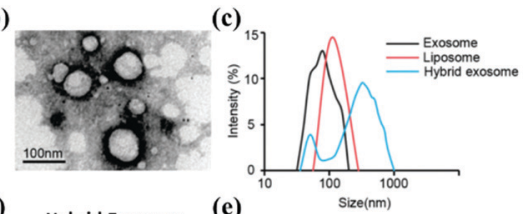

(d)

(e)

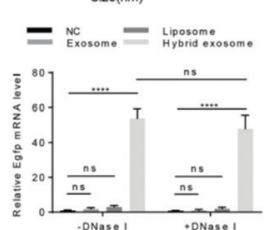

(f)
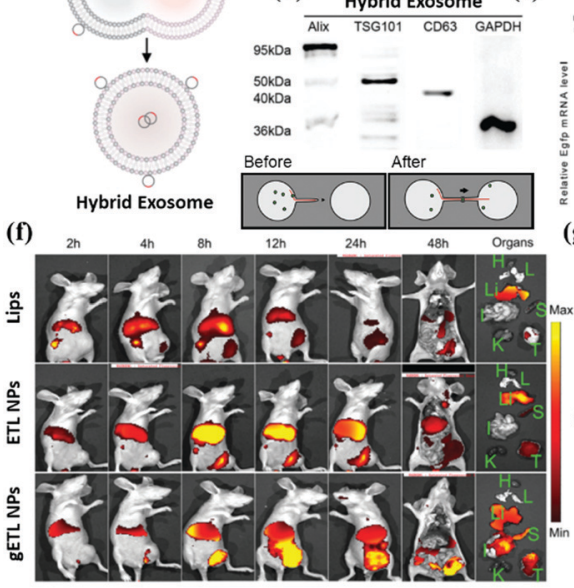

(g)

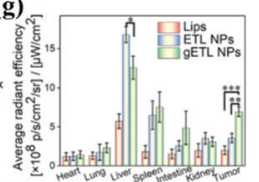

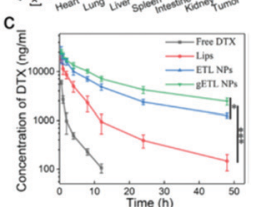

Fig. 5 Assessment of hybrid exosomes encapsulating large plasmids (a) Illustration of the procedure to produce hybrid exosomes. The isolated exosomes were incubated with liposomes for $12 \mathrm{~h}$ at $37{ }^{\circ} \mathrm{C}$ to induce fusion. (b) The combination of exosome and liposome under SEM. (c) DLS measures exosome and liposome and hybrid exosome, respectively. (d) Hybrid Exosome and Exosome have the same surface integral protein. (e) The cargo in the Liposome can be fused with the Exosome so that the Hybrid Exosome contains the target drug or gene. (f) In addition to carrying cargo, the surface modification can also deliver the target molecule or antibody into the hybrid exosome through lipid fusion. (g) Target modification enhances metabolism and enhances therapeutic efficacy. Reproduced with permission. ${ }^{70,71}$ Copyright 2018 and 2019, Wiley Online Library.

loaded with DTX, the results revealed significantly inhibited tumor progression. The anti-tumor effect of HIPEC was enhanced when combined with the treatment (Fig. $5 \mathrm{f}$ and $\mathrm{g}$ ).

In terms of the mechanism, treatment with therapeutic gETL could induce macrophage polarization, enhance macrophage-mediated tumor cell phagocytosis, and induce apoptosis. This study provides a new direction for treating metastatic peritoneal cancer through the combination of drug-loaded gETL and HIPEC.

\subsection{Exosome-coated metal-organic framework nanoparticles}

Metal-organic framework (MOF) materials are an essential classification of new metal-organic materials (MOMs). MOF is one of the most popular research fields in new inorganicorganic materials science because it combines two chemical disciplines, inorganic chemistry and organic chemistry, which are often separated.

MOF consists of metal atoms or clusters of atoms coordinated by organic ligands to form a one-, two-, or three-dimensional structure. ${ }^{94}$ MOF can be used in gas adsorption, gas storage, gas separation, catalysts, and other fields. MOF nanoparticles can form a porous three-dimensional framework. Due to this construction principle, the composition and structure of these materials are highly adjustable. Additionally, the inner and outer surfaces can be independently functionalized. With such characteristics, MOF nanoparticles (NPs) can be designed to meet the specific requirements of the desired application. In the field of drug delivery, a so-called "composite material" with exosomes has been synthesized (Fig. 6a). ${ }^{95}$

Compared with the artificial lipid layer as a closed system, exosomes provide significant advantages: for example, they are non-immunogenic and used by cells for communication purposes. Combined with the high porosity provided by MOF, the composite material can achieve a high drug loading. They are also designed to be biodegradable because they are coated with exosomes. Illes et al. were the first to report that MOF nanoparticles encapsulated by exosomes are an efficient drug delivery system with an "onboard trigger" function (Fig. 6b-e). This approach synergistically combines the characteristics of MOF NPs and exosomes to produce a promising system that promotes easy and efficient loading and sealing. Moreover, it has high treatment efficiency, and medicine does not leak prematurely. ${ }^{77}$ Subsequent articles related to exosome-coated MOF have been published in recent years. ${ }^{96-99}$

A biomimetic nanosystem was designed to assemble cargo proteins in the $\mathrm{pH}$-responsive metal-organic framework (MOF) nanoparticles and use the extracellular vesicle membrane (EVM) to camouflage them. ${ }^{100-102}$ The protein is immobilized in the MOF (ZIF-8) matrix through the self-assembly of the metal node $\left(\mathrm{Zn}^{2+}\right)$ and the blocking of the organic ligand. MOF has a large internal surface area and non-covalent affinity, significantly improving the protein loading efficiency and loading capacity. After the exosome-coated MOF is internalized by the recipient cell, the $\mathrm{pH}$ responsiveness of the metal-ligand bond causes the release of the protein in acidic endosomes and lysosomes. Additionally, the exosome may contain the integrinrelated transmembrane protein $\mathrm{CD} 47$, which mediates a "do not eat me" signal to avoid phagocytosis, allowing the composite material to undergo specific endocytosis. ${ }^{103}$

\subsection{Exosome-coated inorganic nanoparticles}

The development of inorganic nanotechnology has diversified research in chemistry, physics, materials science, engineering, biology, and medicine. Taking biomedicine research as an example, nanomaterials exhibit unique physical and chemical properties at the nanoscale, such as magnetic, optical, thermal, and biomolecule-like properties, which are widely used in biomedical imaging research and development to assist nanomedicine. ${ }^{104}$ Nanomaterials have also been used for diagnosis, treatment, and prevention purposes. In recent years, combining two (or more) functional nanocomposites (bifunctional or multifunctional nanocomposites) has advanced the development of nanomedicine platforms, such as those used to study the growth and aging of the human body, and biochemical manipulation of mechanism and scale has become the driving force for accelerating multimodal imaging. ${ }^{105}$ Next, we enumerate the combinations of common inorganic nanomaterials and exosomes and their related applications in the biomedical field. We briefly describe their 
(a)

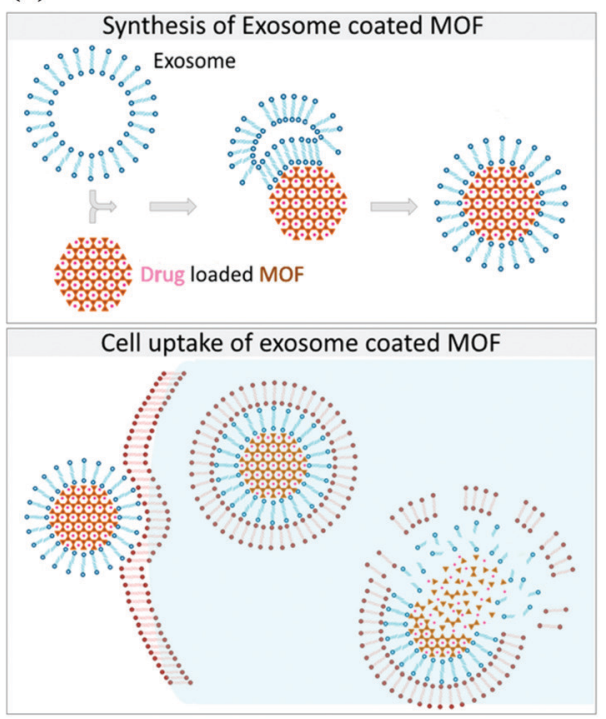

(b)

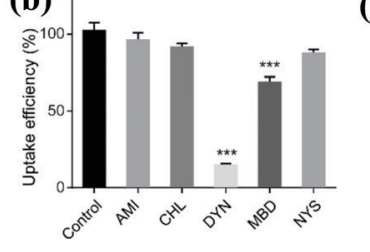

(d)

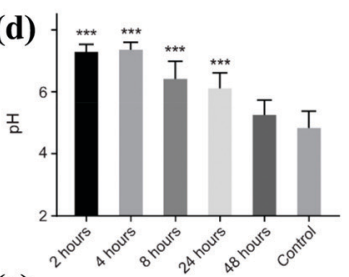

(e)

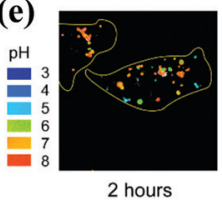

(c)
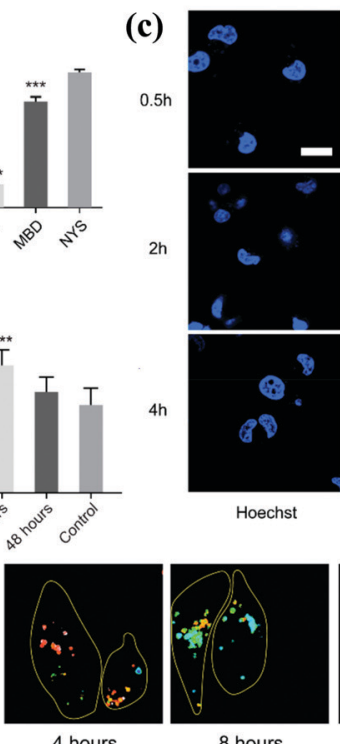

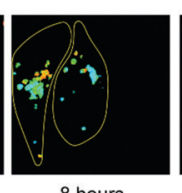

8 hours

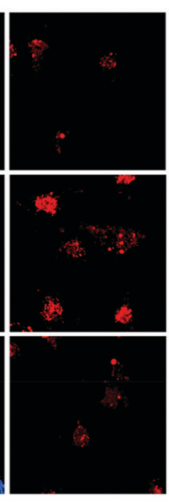

FM4-64

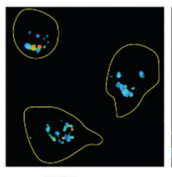

24 hours

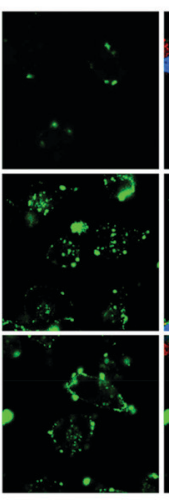

fluoBSA

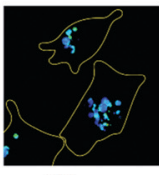

48 hours

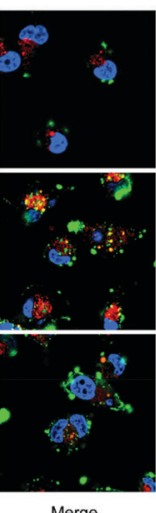

Merge

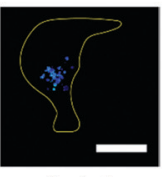

Control

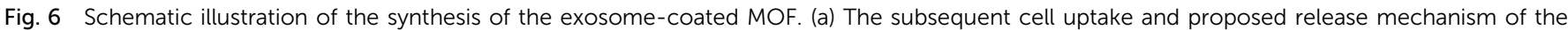

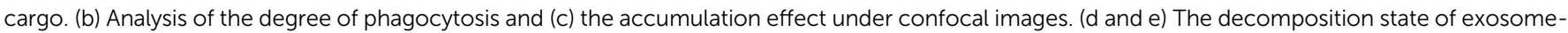
coated MOF under different pH environments. Reproduced with permission. ${ }^{76,77}$ Copyright 2017 and 2018 , American Chemical Society.

applications' current status and prospects in biomedical imaging.

4.3.1. Silica nanoparticles. Nano silicon dioxide is a nonmetallic material that is odorless, non-toxic, and non-polluting. Its particle structure is predominantly network-like, and the basic particle shape is spherical. All silicon dioxide crystals have silicon atoms as the core, and four oxygen atoms are connected to form the tetrahedron. Each oxygen atom is bound to two silicon atoms to create a network structure compound. Nanosilica has high activity and can possess remarkable properties, such as easily modified silanol groups on the surface, optical shielding properties, and chemical stability. ${ }^{106}$ Silica does not react with acids, alkalis, reducing agents, or oxidants at room temperature. Hydrofluoric acid is the leading solution that dissolves the structure of silicon dioxide. The reaction of silicon dioxide with hydrofluoric acid to form $\mathrm{SiF}_{4}$ can detect the content of other substances in silicon dioxide. Silica nanoparticles combined with exosomes can provide the entire nanocomposite with good biocompatibility, prolong circulation, and tumor targeting capacity through the specific protein on the lipid-based structure of the exosome. In addition, the exosome shows effective cellular uptake and targeted homing ability, which depends on the particular protein composition on their membranes. Since the surface protein composition of exosomes may be critical to its function, maintaining the integrity and stability of the exosomal membrane is very important for its application in drug delivery. In recent years, neatly arranged mesopores have been prepared by exploiting the self-assembly capabilities of different surfactants; thus, mesoporous materials have received significant attention. Their properties include a stable and consistent pore structure, high surface area, adjustable pore size, precise and adaptable surface properties, non-toxicity, and good biocompatibility. ${ }^{107}$
At present, many published reports describe using mesoporous silicon materials or modified mesoporous silicon materials as carriers, along with their applications in drug delivery systems. Yong et al. proposed using porous silicon nanoparticles (PSiNPs) as drug carriers to deliver into tumor cells and generate PSiNPs (E-PSiNPs) through exosomes as a drug carrier for targeted cancer chemotherapy. When tumor cells are incubated with doxorubicin-loaded PSiNPs (DOX@ PSiNPs), they will pass by the exocytotic pathway to generate exosome-encapsulated DOX-loaded PSiNPs (DOX@E-PSiNPs). DOX@E-PSiNPs also possess the characteristics of exosomal targeting, drug loading, and biological diagnostics, which leads to applying biomimetic nanoparticles as drug carriers to effectively deliver anticancer drugs (Fig. 7a). The biodistribution evaluation also demonstrates E-PSiNPs can be used as a targeting flatform for drug accumulation (Fig. 7b). ${ }^{108,109}$ In addition to their drug delivery functions, mesoporous materials can be modified through functional groups on their surfaces to provide them with different properties, which can be applied to various types of biomedicine and biotechnology. For example, cell type identification, disease diagnosis, intracellular visualization, and drug and gene delivery have been researched, and results confirm that mesoporous materials can enter cells through endocytosis (Fig. 7c). The authors also revealed that E-PSiNPs could send the whole nanosystem to enter the core of cells (Fig. 7d). These grafted molecules can use various environmental factors, such as light-cutting effects, redox reactions, the presence of enzymes, or different $\mathrm{pH}$ values, to produce different chemical reactions, and then control the opening and closing of holes to achieve the effect of controlled drug release (Fig. 7e and f).

4.3.2. Quantum dots. Fluorescent nanomaterials have become widely used in biological applications, including biosensors, drug delivery, and bioimaging. ${ }^{110}$ The development 

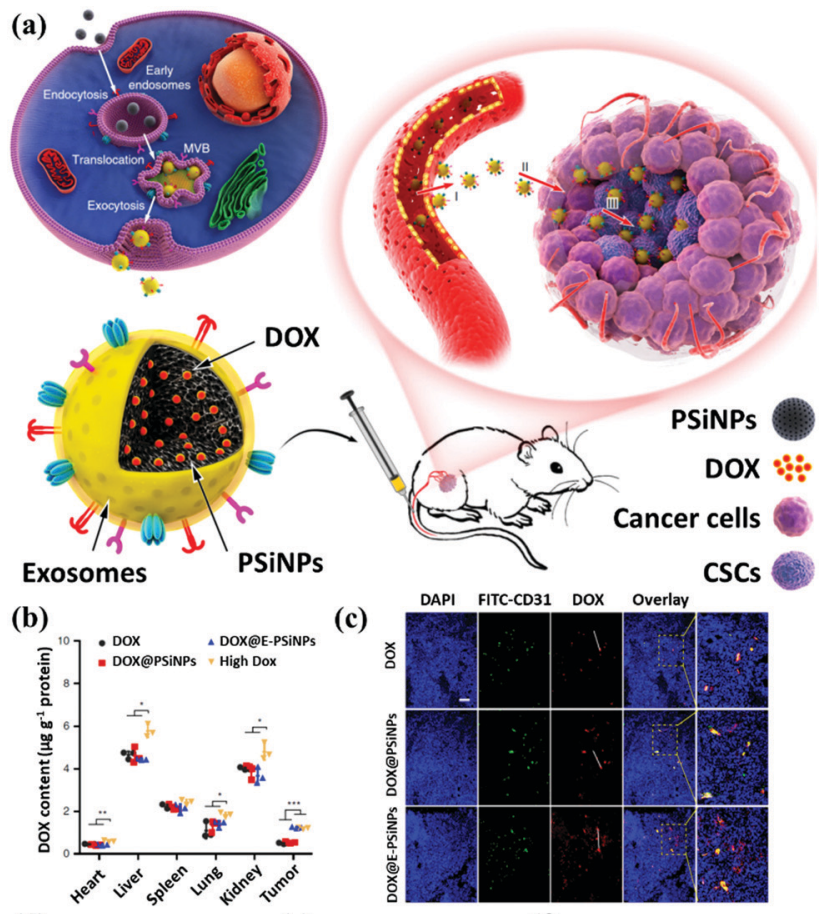

(c)
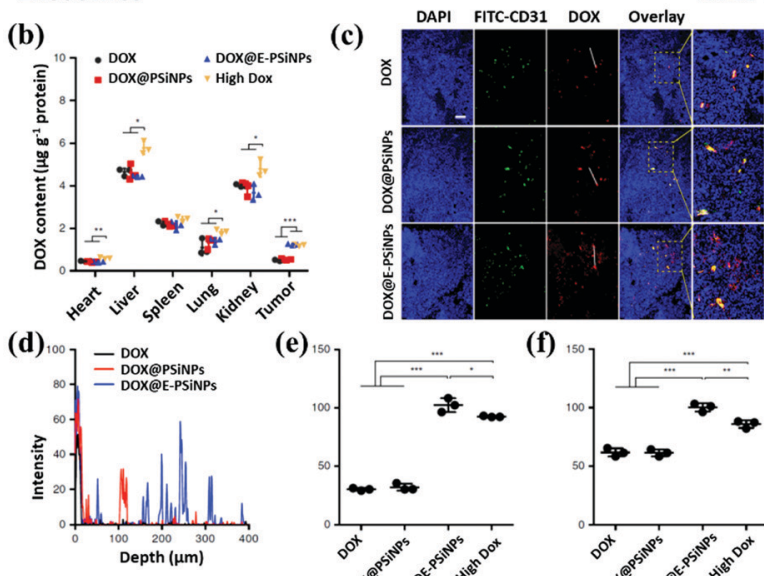

(e)
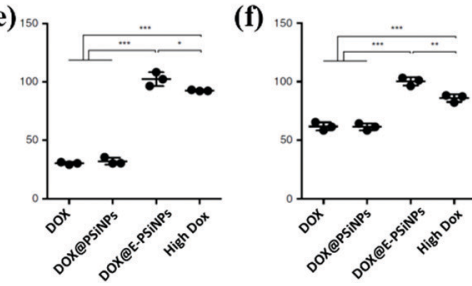

Fig. 7 Schematic illustration of E-PSiNPs as drug carriers for targeted cancer chemotherapy. (a) Schematic illustration of the preparation of DOX@E-PSiNPs. DOX@PSiNPs are endocytosed into cancer cells after incubation and localized in multivesicular bodies (MVBs) and autophagosomes. After MVBs or amphisomes fuse with the cell membrane, DOX@EPSiNPs are exocytosed into extracellular space. (b) Schematics showing how DOX@E-PSiNPs efficiently target tumor cells after intravenous injection into tumor-bearing mice. (c) The confocal images and (d) signal quantification of DOX(aE-PSiNPs accumulation. The DOX accumulation under (e) neutral and (f) low $\mathrm{pH}$ values. Reproduced with permission. ${ }^{78}$ Copyright 2019, Springer Nature Limited.

of fluorescent nanomaterials is of great interest to scientists; these materials are classified as conductors (gold nanoclusters), insulators (upconversion nanoparticles), and semiconductors (quantum dots and carbon dots) based on different conductivities. Among these materials, quantum dots (QDs) have undergone rapid growth because of the tunable emission range from the blue to near-infrared region due to quantum confinement, narrow full width at half maximum (FWHM), and high photostability. ${ }^{111}$ Based on various compositions of QDs, QD nanomaterials can be divided into four classes: II-VI, IV, III-V, and I-III- $\mathrm{VI}_{2}$ QDs. I-III-VI ${ }_{2}$ ternary QDs are fabricated by two cations $\left(\mathrm{I}=\mathrm{Cu}^{+}\right.$and $\mathrm{Ag}^{+} ; \mathrm{III}=\mathrm{Al}^{3+}, \mathrm{Ga}^{3+}$, or $\left.\mathrm{In}^{3+}\right)$ and one anion $\left(\mathrm{VI}=\mathrm{S}^{2-}, \mathrm{Se}^{2-}\right.$, or $\left.\mathrm{Te}^{2-}\right) .{ }^{112}$ Lannoo et al. used QDs as a model to investigate the influence of the quantum confinement effect, which results in an energy gap between the valence band and the conduction band. Eqn (1-1) reflects the relationship between the change in size and the energy gap. ${ }^{113}$

$$
E_{\mathrm{n}}=E_{\mathrm{g}}+\frac{h^{2} \pi^{2}}{2 \mu R^{2}} .
$$

$E_{\mathrm{n}}$ : energy gap of nanomaterials; $E_{\mathrm{g}}$ : energy gap of bulk materials; $\mu$ : electron-hole pair condensation of quality; $h$ : Planck's constant; $R$ : radius of the nanomaterials.

As illustrated in the above formula, the energy gap of nanomaterials is inversely proportional to their size. In modern biological analysis, various kinds of organic dyes are used. However, as technology advances, greater flexibility in these dyes is being sought.

To this end, quantum dots have quickly filled this role, as they are superior to traditional organic dyes on several counts, with brightness being among the most immediately obvious (owing to the high extinction coefficient combined with a comparable quantum yield to fluorescent dyes) as well as their stability (leading to much less photobleaching). It has been estimated that quantum dots are 20 times brighter and 100 times more stable than traditional fluorescent reporters. For single-particle tracking, the irregular blinking of quantum dots is a minor drawback. However, several groups have developed quantum dots that are essentially nonblinking. Their utility in single-molecule tracking experiments has been demonstrated. ${ }^{114,115}$ However, the toxicity and stability of quantum dots are still shortcomings that need to be overcome in current research. Liu et al. proposed a method of coating exosomes on the periphery of black phosphorus quantum dots (BPQDs). The author first took out the mouse blood to purify the exosome, and then added BPQDs to the exosome (Fig. 8a). Quantum dots with exosomes on the surface have better dispersion than simple quantum dots (Fig. 8b and f). Next, the thickness and element distribution of the material was analyzed through an atomic force microscope (Fig. 8c-e), confirming that the size of the material is close to the same, reflecting the potential strength of exosome to optimize nanomaterials. Then analyze the material size (Fig. 8g) and the total BPQDs carried by the exosome (Fig. 8h). Finally, to confirm that the outer layer is an exosome membrane, a western blot was used to analyze the markers of the composite material (Fig. 8i).

4.3.3. Transition elements nanoparticles. The electronic configurations of transition elements are rich and diverse. When there is a single electron in the electronic orbital of the transition element, the resulting complex can provide the material with different magnetic properties. For example, iron metal has a variety of metal ions that exist stably, exhibit ferromagnetic properties, and even has superparamagnetism at the nanometer size. The manganese family is particularly prominent in the single-electron valence state, with the $\mathrm{d}^{5}$ configuration. It can form coordination with various elements, such as MnCO. Since transition metals have variable valence states, they usually exist in the natural environment in several different stable oxidation states, such as $\mathrm{Fe}_{3} \mathrm{O}_{4}$.

In addition, the electron-ion pairs in these elements can correspond to the absorption of photons in the visible electromagnetic spectrum, resulting in colored compounds. Due to 
(a)

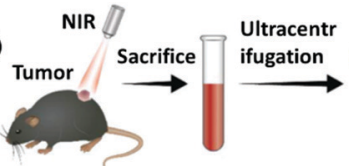

NIR-treated mice Blood

(b)

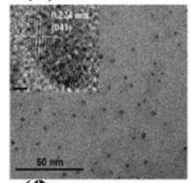

(f)

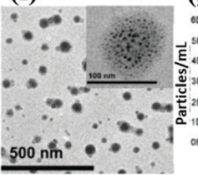
(c)

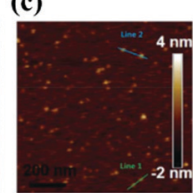

(g)

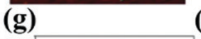

(h)

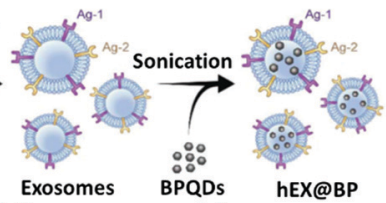

(d)

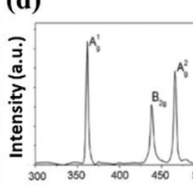

(e)

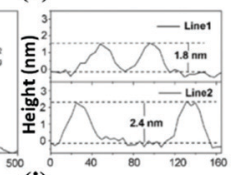

(i)
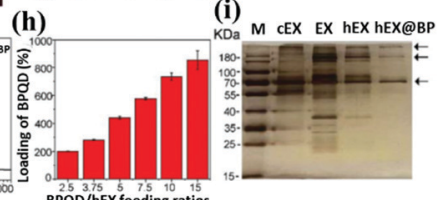

Fig. 8 Schematic representation of black phosphorus quantum dots through exosome coating. (a) Graphic illustration describes the structure of exosome-encapsulated black phosphorus quantum dots (hEX@BP) and their roles in photothermal immunotherapy. Ag: antigen; NIR: near-infrared BPQDs: black phosphorus quantum dots. Transmission electron microscopy images of (b) BPQDs and (f) hEX@BP. The atomic force microscope indicated the (c) linear depth, (d) Raman shift, and (e) height of BPs. The (g) size distribution, (h) BPQDs loading ratio, and (i) western blot of hEX@BP. Reproduced with permission. ${ }^{79}$ Copyright 2020 , Royal Society of Chemistry.

these behaviors, transition metals exhibit rich and fascinating chemical properties. In 2021, Pan et al. designed a nanocarrier based on the exosome-embedded and dox-loaded phosphomolybdic acid/ $\mathrm{Fe}_{3} \mathrm{O}_{4}$ nanoparticles (Exo-PMA/Fe-HSA@DOX, Fig. 9a). ${ }^{80}$ They use the urinary exosomal membrane as a nano-platform for chemical/chemokinetic therapy and targeted homogenous prostate cancer diagnosis. The nanocarriers of urinary exosomes do not contain intracellular substances such as histone 3 and COX IV (Fig. 9b). Successfully achieved excellent synergistic low-dose chemical/chemical kinetic performance in vivo. In addition, the increased intracellular PMA/Fe-HSA@DOX nanoparticles (NPs) greatly enhance the bypass of the EGFR/AKT/NF-kB/IkB signaling pathway. The in vivo mice model is used to verify its therapeutic effect, which shows that the targeted homologous cancer therapy based on the urine exosomes of cancer patients embodies a new type of targeted anticancer program in an efficient and straightforward method (Fig. 9c). In the same year, Zhu et al. developed exosomal nanovesicles (MMV) loaded with manganese carbonyl (MnCO) to overcome this tumor treatment problem (Fig. 9d). ${ }^{82}$ MMV has a uniform size and good dispersion (Fig. 9e and f). After administration, MMV has shown admirable performance in actively targeting tumors, mitochondrial damage, and radiosensitization therapy and can promote $90 \%$ tumor growth inhibition under very low dose (only 2Gy) radiotherapy (Fig. 9g). Overall, this study emphasizes a novel and practical way to improve the efficacy of tumor radiotherapy.

4.3.4. Gold nanoparticles. Gold nanoparticles, one of the noble metal nanomaterials, are the most well-known nanomaterial in colloidal solutions. In 2009, Palui et al. attributed the red color of matter to $\mathrm{Au}$ nanoparticles, and he synthesized this nanomaterial using a wet chemical synthesis method. ${ }^{116}$ Since then, gold

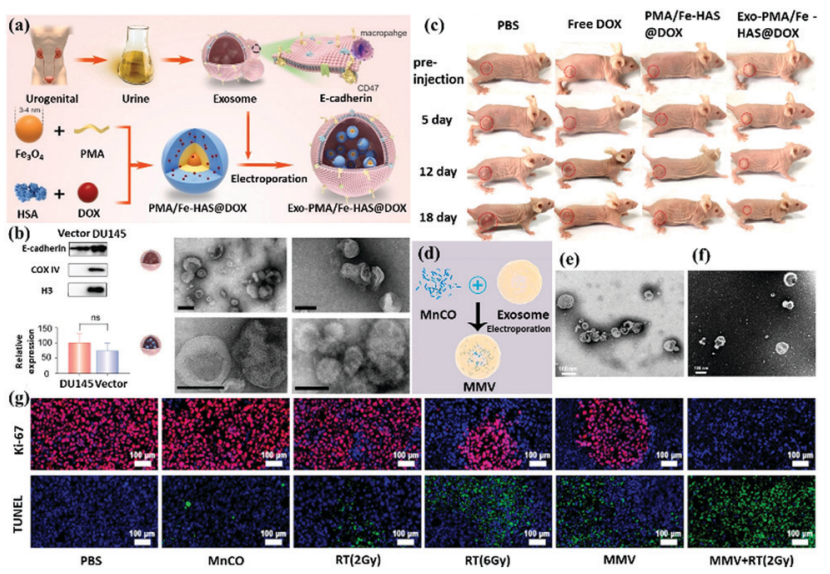

Fig. 9 Schematic representation of transition elements nanoparticles $\left(\mathrm{Fe}_{3} \mathrm{O}_{4}\right.$ and $\left.\mathrm{MnCO}\right)$ functionalization through exosome coating. (a) Preparation method and synthesis schematic diagram of Exo-PMA/ $\mathrm{Fe}_{3} \mathrm{O}_{4}$. (b) The performance of the vector in exosomes and the changes in $\mathrm{pH}$ environment regulation morphology of Exo- $\mathrm{PMA} / \mathrm{Fe}_{3} \mathrm{O}_{4}$. (c) Analysis of mouse treatment data. (d) Schematic diagram of MnCO@Exosome (MMV) preparation. (e) TEM and (f) SEM topography analysis. (g) Tissue staining analysis and use radiotherapy together with MMV for treatment. Reproduced with permission. ${ }^{80,82}$ Copyright 2021, Elsevier Ltd. All.

nanoparticles have been a popular material because of their unique optical properties, resulting from their localized surface plasmon resonance (LSPR). ${ }^{117}$ LSPR occurs at the nanoscale because the incident light is far more significant than the nanomaterials, and as a result, all free electrons on the surface react together.

This unique physical phenomenon occurs only for metallic elements with an abundance of free conductive electrons that are easily affected by irradiating light, for example, $\mathrm{Au}, \mathrm{Ag}$, and $\mathrm{Cu}$. The SPR effect for metallic colloid nanoparticles displays a unique physical impact and corresponds to the color of the solution. For example, $13 \mathrm{~nm}$ gold nanoparticles exhibit a red color in solution because their LSPR peak is $520 \mathrm{~nm}$. The wavelength of SPR in the previously mentioned study can be derived from eqn (1-2), as shown below. ${ }^{118}$ The SPR frequency $\omega_{\text {sp }}$ of metal nanoparticles is related to their geometry factor $\kappa$ and the dielectric constant $\varepsilon_{\mathrm{m}}$ of the surrounding medium. Wavelength and frequency are reciprocally related.

$$
\omega_{\mathrm{sp}}=\sqrt{\frac{\mathrm{ne}^{2}}{m_{0} \varepsilon_{0}\left(\varepsilon_{\infty}+k \varepsilon_{\mathrm{m}}\right)}-\Gamma^{2}} .
$$

$\omega_{\mathrm{sp}}$ : surface plasmon resonance wavelength; $n$ : free carrier density; $m_{0}$ : the effective mass of charge carrier; $\varepsilon_{0}$ : dialectic constant of free space; $\varepsilon_{\mathrm{m}}$ : medium dielectric constant; $\varepsilon_{\infty}$ : high dielectric constant of the mediuml; $k$ : geometry of nanomaterials; $\Gamma$ : damping constant.

From this equation and the literature, the most convenient factor for changing the peak site of SPR is the geometry factor. ${ }^{119}$ For gold nanoparticles of various sizes, the SPR peak slightly shifts from about 520 to $540 \mathrm{~nm}$ as the particle size increases from 5 to $100 \mathrm{~nm}$.

Therefore, the geometric structures of gold nanorods, gold nanoshells, and gold nanocages are commonly used to 


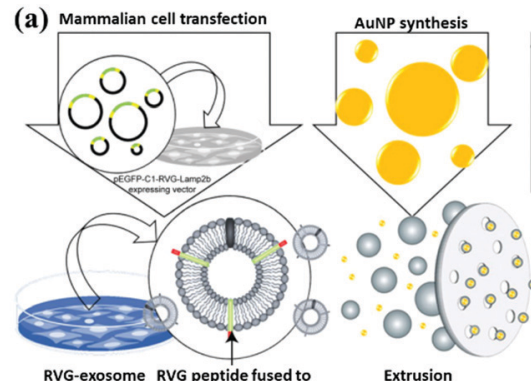

RVG-exosome RVG peptide fused to
isolation Lamp2b protein

(b)

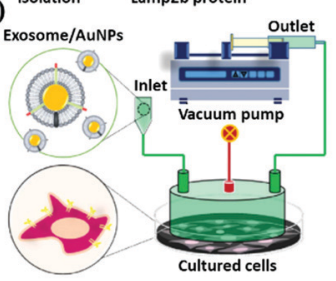

(c)

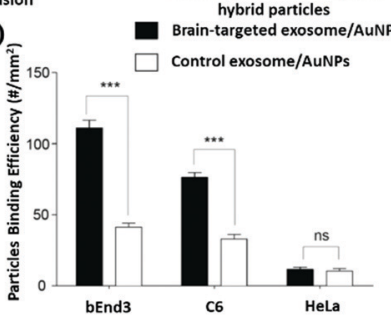

(d)

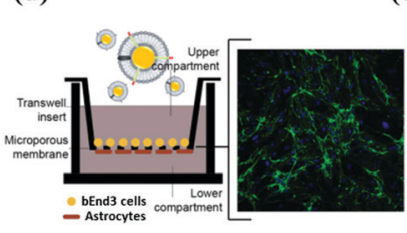

(e)

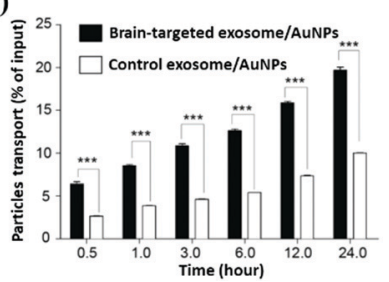

Fig. 10 Schematic representation of gold nanoparticle surface functionalization through exosome coating. (a) The brain-targeting property of AuNPs after fabrication with neuron-targeted exosomes. Signals of fluorescently labeled nanoparticles in the basal chamber were measured in $0.5 \mathrm{~mL}$ aliquots at different time points. (b) A microinjection device was used to deliver exosome/AuNPs systematically. (c) Quantification of exosome/AuNPs and determining the degree of phagocytosis of the material by various cell lines. Confocal microscope (d) image analysis of cell uptake and (e) quantifying the endocytosis effect of cells on exosome/AuNPs under transwell incubation. Reproduced with permission. ${ }^{85}$ Copyright 2018, Royal Society of Chemistry.

determine the difference in SPR peaks. Based on the SPR effect of the gold nanomaterial, Sancho-Albero et al. proposed a technique of exosome embedding gold nanoparticles. In Fig. 10a, the exosome is first released from the cells. The medium is extracted to purify the exosome, which is mixed with gold nanoparticles and fabricated to produce a gold nanoparticle surface-functionalized exosome. Moreover, the authors tested different cell lines and confirmed that exosomes could lead gold nanoparticles to target and accumulate in the cancer cells after pumping the exosome/AuNPs (Fig. 10b). To quantify the accumulation effect of exosome/AuNPs, they chose different cell lines to show that exosomes can actively carry AuNPs into the cancer cells (Fig. 10c). The gold nanoparticles are sent into the cells through transwell co-culture at different time points for Confocal (Fig. 10d) and Time course (Fig. 10e) analysis. ${ }^{85}$

\section{Conclusions and future perspectives}

Recent studies have found that exosomes or EVs are associated with cancer metastasis, disease progression, diagnosis, and

treatment, leading to a rapidly emerging cell research area. Cells secrete exosomes via exocytosis. This has also been observed for other rigid nanoparticles, such as silica and carbon. Relying on the superior targeting function and biocompatibility of cancer exosomes, researchers have been able to deliver drugs to cancer stem cells deep within tumors in mouse models. Despite significant efforts made in this relatively new field of exosome research, progress has been held back by challenges such as inefficient separation methods, difficulties in characterization, and a lack of specific biomarkers.

Moreover, the applications of exosomes need to be further evaluated under actual physiological conditions. Inorganic/ organic nanoparticles, on the other hand, produce powerful in vivo near-infrared fluorescence, thus generating remarkable tumor imaging tracking ability in subcutaneous, orthotopic, and metastatic tumors. This review describes an approach to cancer therapy that uses exosome-biomimetic nanoparticles. Hybrid inorganic/organic nanoparticles with exosomes can be applied as drug carriers to deliver anticancer drugs to the centers of cancer cells efficiently. Biocompatible hybrid inorganic/organic nanoparticles with exosomes have been synthesized for targeted cancer chemotherapy. Following intravenous injection, nanocomposites actively accumulate in the tumor site. They are subject to cross-reactive cellular uptake by cancer cells, resulting in augmented in vivo drug delivery enrichment in tumor cells. At present, diversified hybrid inorganic/organic nanoparticles with exosomes for both therapy and diagnosis are still in urgent need of development.

This combination can achieve theranostics on the same platform and provide advantages such as targeting, improved dispersion, and avoiding immune system clearance. However, as in most clinical applications, the biological application of nanomaterials still needs more research to optimize and improve their performance in vivo. The complexity of hybrid inorganic/organic nanoparticles with exosomes will require more attention to their safety and biocompatibility, even though they may provide excellent therapeutic and diagnostic functions. In addition, its biodegradability also needs to be evaluated to ensure that the inorganic/organic materials will not accumulate in the body. Therefore, it is believed that hybrid inorganic/organic nanoparticles with exosomes will become a hot topic for future research after being baptized by scientists' brainstorming.

\section{Author contributions}

Conceptualization, M. H., R. S. L., L. J. L., and C. Y. H.; writing original draft preparation, M.-H. C. and Z.-X. C.; writing review and editing, M. H. C. and Z. X. C.; supervision, R. S. L. and $\mathrm{M}$. H. All authors have read and agreed to the published version of the manuscript.

\section{Conflicts of interest}

There are no conflicts to declare. 


\section{Acknowledgements}

This research was supported by Genomics Research Center, Academia Sinica, Taiwan to M. H. and by Ministry of Science and Technology (MOST 109-2113-M-002-020-MY3) to R. S. L. In addition, Ming-Hsien Chan is supported by the Academia Sinica Outstanding Postdoctoral Fellowship.

\section{References}

1 A. V. Vlassov, S. Magdaleno, R. Setterquist and R. Conrad, Biochim. Biophys. Acta, Gen. Subj., 2012, 1820, 940-948.

2 S. A. A. Kooijmans, P. Vader, S. M. van Dommelen, W. W. van Solinge and R. M. Schiffelers, Int. J. Nanomed., 2012, 7, 1525-1541.

3 D. Ha, N. N. Yang and V. Nadithe, Acta Pharm. Sin. B, 2016, 6, 287-296.

4 H. Valadi, K. Ekstrom, A. Bossios, M. Sjostrand, J. J. Lee and J. O. Lotvall, Nat. Cell Biol., 2007, 9, 654-672.

5 H. Q. Xin, Y. Li and M. Chopp, Front. Cell. Neurosci., 2014, 8, 377 .

6 P. A. Steck, M. A. Pershouse, S. A. Jasser, W. K. A. Yung, H. Lin, A. H. Ligon, L. A. Langford, M. L. Baumgard, T. Hattier, T. Davis, C. Frye, R. Hu, B. Swedlund, D. H. F. Teng and S. V. Tavtigian, Nat. Genet., 1997, 15, 356-362.

7 D. Szkutnik-Fiedler, Pharmaceutics, 2020, 12, 1180.

8 C. C. Chen, L. N. Liu, F. X. Ma, C. W. Wong, X. N. E. Guo, J. V. Chacko, H. P. Farhoodi, S. X. Zhang, J. Zimak, A. Segaliny, M. Riazifar, V. Pham, M. A. Digman, E. J. Pone and W. A. Zhao, Cell. Mol. Bioeng., 2016, 9, 509-529.

9 S. Kamerkar, V. S. LeBleu, H. Sugimoto, S. J. Yang, C. F. Ruivo, S. A. Melo, J. J. Lee and R. Kalluri, Nature, 2017, 546, 498-499.

10 S. G. Antimisiaris, S. Mourtas and A. Marazioti, Pharmaceutics, 2018, 10, 218.

11 L. Song, P. P. Li, W. Yang, X. H. Lin, H. Liang, X. F. Chen, G. Liu, J. Li and H. H. Yang, Adv. Funct. Mater., 2018, 28, 1707496.

12 J. Y. Ha, S. Y. Choi, J. H. Lee, S. H. Hong and H. J. Lee, Front Mol. Biosci., 2020, 7, 596366.

13 R. C. Lai, R. W. Y. Yeo, K. H. Tan and S. K. Lim, Biotechnol. Adv., 2013, 31, 543-551.

14 C. Schindler, A. Collinson, C. Matthews, A. Pointon, L. Jenkinson, R. R. Minter, T. J. Vaughan and N. J. Tigue, PLoS One, 2019, 14, e0214545.

15 M. P. Stewart, A. Sharei, X. Y. Ding, G. Sahay, R. Langer and K. F. Jensen, Nature, 2016, 538, 183-192.

16 A. Calistri, P. Sette, C. Salata, E. Cancellotti, C. Forghieri, A. Cornin, H. Gottlinger, G. Campadelli-Fiume, G. Palu and C. Parolin, J. Virol., 2007, 81, 11468-11478.

17 R. M. Johnstone, M. Adam, J. R. Hammond, L. Orr and C. Turbide, J. Biol. Chem., 1987, 262, 9412-9420.

18 J. Kowal, M. Tkach and C. Thery, Curr. Opin. Cell Biol., 2014, 29, 116-125.
19 N. P. Hessvik and A. Llorente, Cell. Mol. Life Sci., 2018, 75, 193-208.

20 Y. Q. Koh, F. B. Almughlliq, K. Vaswani, H. N. Peiris and M. D. Mitchell, Front. Biosci.-Landmark., 2018, 23, 865-874.

21 F. Yang, X. Z. Liao, Y. Tian and G. Y. Li, Biotechnol. J., 2017, 12, 1600699.

22 J. Lee, H. Kim, Y. Heo, Y. K. Yoo, S. I. Han, C. Kim, D. Hur, H. Kim, J. Y. Kang and J. H. Lee, Analyst, 2020, 145, 157-164.

23 S. S. Huang, L. Wang, T. F. Bruce and R. K. Marcus, Biotechnol. Prog., 2020, 36, e2998.

24 P. Saravanan, J. Gu, X. Wang, P. Nguyen, G. Yoshimatsu, M. Lawrence and B. Naziruddin, Am. J. Transplant., 2017, 17, 723.

25 Y. A. Bai, Y. X. Lu, K. Wang, Z. L. Cheng, Y. L. Qu, S. H. Qiu, L. Zhou, Z. H. Wu, H. Y. Liu, J. L. Zhao and H. L. Mao, Nano-Micro Lett., 2019, 11, 59.

26 Y. Z. Zhu, S. Lee, X. R. Zheng, G. J. Zheng, H. R. Tseng and S. Hou, Mod. Pathol., 2017, 30, 538a.

27 C. Y. Soo, Y. Q. Song, Y. Zheng, E. C. Campbell, A. C. Riches, F. Gunn-Moore and S. J. Powis, Immunology, 2012, 136, 192-197.

28 C. Gardiner, R. Dragovic, D. Tannetta, P. Harrison, C. Redman and I. Sargent, Int. J. Lab. Hematol., 2012, 34, 87.

29 E. van der Pol, F. A. W. Coumans, A. E. Grootemaat, C. Gardiner, I. L. Sargent, P. Harrison, A. Sturk, T. G. van Leeuwen and R. Nieuwland, J. Thromb. Haemostasis, 2014, 12, 1182-1192.

30 K. Kluszczynska, L. Czernek, W. Cypryk, L. Peczek and M. Duchler, Curr. Pharm. Des., 2019, 25, 4464-4485.

31 M. Catalano and L. O'Driscoll, J. Extracell. Vesicles, 2020, 9, 1703244.

32 Y. Zheng, A. Hasan, M. M. Nejadi Babadaei, E. Behzadi, M. Nouri, M. Sharifi and M. Falahati, Biomed. Pharmacother., 2020, 129, 110442.

33 X. Y. Zhao, D. L. Wu, X. D. Ma, J. L. Wang, W. J. Hou and W. Zhang, Biomed. Pharmacother., 2020, 128, 110237.

34 Z. Wan, Y. Dong, M. Y. Wei, X. T. Gao, G. D. Yang, J. Zhang and L. Liu, Adv. Biosyst., 2020, 4, e2000061.

35 J. E. Pullan, M. I. Confeld, J. K. Osborn, J. Kim, K. Sarkar and S. Mallik, Mol. Pharmaceutics, 2019, 16, 1789-1798.

36 G. Morales, D. Cardenas, C. Arroyo, L. Ayala, R. Seqqat and M. Torres, Trop. Med. Int. Health, 2017, 22, 129-130.

37 Y. H. Chang, K. C. Wu, H. J. Harn, S. Z. Lin and D. C. Ding, Cell Transplant., 2018, 27, 349-363.

38 R. Pluta and M. Ułamek-Kozioł, Adv. Exp. Med. Biol., 2019, 1118, 71-82.

39 L. Q. Jiang, H. J. Dong, H. Cao, X. F. Ji, S. Y. Luan and L. Liu, Med. Sci. Monit., 2019, 25, 3329-3335.

40 X. B. Qin, S. R. Yu, L. L. Zhou, M. Q. Shi, Y. Hu, X. Y. Xu, B. Shen, S. W. Liu, D. L. Yan and J. F. Feng, Int. J. Nanomed., 2017, 12, 3721-3733.

41 D. L. Yuwen, B. B. Sheng, J. Liu, W. Wenyu and Y. Q. Shu, Eur. Rev. Med. Pharmaco., 2017, 21, 2650-2658.

42 W. J. Huang, Y. Y. Yan, Y. Liu, M. T. Lin, J. X. Ma, W. Zhang, J. W. Dai, J. J. Li, Q. R. Guo, H. B. Chen, 
B. Makabel, H. Liu, C. Y. Su, H. Bi and J. Y. Zhang, Signal Transduction Targeted Ther., 2020, 5, 39.

43 Y. T. Wang, T. J. Shi, S. Srivastava, J. Kagan, T. Liu and K. D. Rodland, Cancers, 2020, 12, 2335.

44 N. Wang, X. G. Song, L. S. Liu, L. M. Niu, X. W. Wang, X. R. Song and L. Xie, Cancer Sci., 2018, 109, 1701-1709.

45 D. J. Clark, W. E. Fondrie, A. Yang and L. Mao, J. Proteomics, 2016, 133, 161-169.

46 K. O’Brien, K. Breyne, S. Ughetto, L. C. Laurent and X. O. Breakefield, Nat. Rev. Mol. Cell Biol., 2020, 21, 585-606.

47 J. Guan, Q. Shen, Z. Zhang, Z. X. Jiang, Y. Yang, M. Q. Lou, J. Qian, W. Y. Lu and C. Y. Zhan, Nat. Commun., 2018, 9, 2982.

48 T. Yamashita, Y. Takahashi, M. Nishikawa and Y. Takakura, Eur. J. Pharm. Biopharm., 2016, 98, 1-8.

49 A. Thakur, R. K. Sidu, H. Zou, M. K. Alam, M. S. Yang and Y. J. Lee, Int. J. Nanomed., 2020, 15, 8331-8343.

50 X. M. Guo, J. Ma, J. Sun and G. X. Gao, Proc. Natl. Acad. Sci. U. S. A., 2007, 104, 151-156.

51 D. Gallego-Perez, D. Pal, S. Ghatak, V. Malkoc, N. HiguitaCastro, S. Gnyawali, L. Q. Chang, W. C. Liao, J. F. Shi, M. Sinha, K. Singh, E. Steen, A. Sunyecz, R. Stewart, J. Moore, T. Ziebro, R. G. Northcutt, M. Homsy, P. Bertani, W. Lu, S. Roy, S. Khanna, C. Rink, V. B. Sundaresan, J. J. Otero, L. J. Lee and C. K. Sen, Nat. Nanotechnol., 2017, 12, 974-979.

52 L. A. Ambattu, S. Ramesan, C. Dekiwadia, E. Hanssen, H. Y. Li and L. S. Y. Yeo, Commun. Biol., 2020, 3, 553.

53 S. W. Guo, N. Perets, O. Betzer, S. Ben-Shaul, A. Sheinin, I. Michaelevski, R. Popovtzer, D. Offen and S. Levenberg, ACS Nano, 2019, 13, 10015-10028.

54 M. Kim, G. Kim, D. W. Hwang and M. Lee, J. Biomed. Nanotechnol., 2019, 15, 2401-2412.

55 H. Y. Tao, H. L. Xu, L. Zuo, C. Li, G. Qiao, M. Y. Guo, L. H. Zheng, M. Leitgeb and X. K. Lin, Int. J. Biol. Macromol., 2020, 161, 470-480.

56 Y. H. Cao, E. B. Ma, S. Cestellos-Blanco, B. Zhang, R. Y. Qiu, Y. D. Su, J. A. Doudna and P. D. Yang, Proc. Natl. Acad. Sci. U. S. A., 2019, 116, 7899-7904.

57 Z. J. Xu, S. S. Zeng, Z. C. Gong and Y. L. Yan, Mol. Cancer, 2020, 19, 160.

58 A. Gallo, S. Vella, M. Miele, F. Timoneri, M. Di Bella, S. Bosi, M. Sciveres and P. G. Conaldi, Cancer Lett., 2017, 388, 334-343.

59 M. Iero, R. Valenti, V. Huber, P. Filipazzi, G. Parmiani, S. Fais and L. Rivoltini, Cell Death Differ., 2008, 15, 80-88.

60 H. Y. Xu, N. Li, N. Yao, X. F. Xu, H. X. Wang, X. Y. Liu and Y. Zhang, Oncol. Rep., 2019, 42, 866-879.

61 L. H. Lv, Y. L. Wan, Y. Lin, W. Zhang, M. Yang, G. L. Li, H. M. Lin, C. Z. Shang, Y. J. Chen and J. Min, J. Biol. Chem., 2012, 287, 15874-15885.

62 H. Samaha, A. Pignata, K. Fousek, J. Ren, F. W. Lam, F. Stossi, J. Dubrulle, V. S. Salsman, S. Krishnan, S. H. Hong, M. L. Baker, A. Shree, A. Z. Gad, T. Shum, D. Fukumura, T. T. Byrd, M. Mukherjee, S. P. Marrelli,
J. S. Orange, S. K. Joseph, P. H. Sorensen, M. D. Taylor, M. Hegde, M. Mamonkin, R. K. Jain, S. El-Naggar and N. Ahmed, Nature, 2018, 561, 331-332.

63 X. J. Gao, N. Ran, X. Dong, B. F. Zuo, R. Yang, Q. B. Zhou, H. M. Moulton, Y. Q. Seow and H. F. Yin, Sci. Transl. Med., 2018, 10, eaat0195.

64 B. H. Liang, X. M. Hu, Y. H. Ding and M. J. Liu, J. Cell. Physiol., 2021, 236, 4138-4151.

65 M. Naseri, M. Bozorgmehr, M. Zoller, E. R. Pirmardan and Z. Madjd, OncoImmunology, 2020, 9, 1779991.

66 N. Abu, N. Othman, N. S. Ab Razak, S. N. Nasir and R. Jamal, Gut, 2019, 68, A14-A15.

67 V. S. LeBleu and R. Kalluri, Immunity, 2019, 50, 547-549.

68 X. Y. Qiu, J. Liu, C. X. Zheng, Y. T. Su, L. L. Bao, B. Zhu, S. Y. Liu, L. L. Wang, X. Wang, Y. R. Wang, W. M. Zhao, J. Zhou, Z. H. Deng, S. Y. Liu and Y. Jin, Cell Proliferation, 2020, 53, e12830.

69 S. Dusoswa, S. Horrevorts, S. Schetters, T. Wurdinger, Y. Van Kooyk and J. Garcia-Vallejo, Neuro-Oncology, 2018, 20, 89.

70 Y. Lin, J. H. Wu, W. H. Gu, Y. L. Huang, Z. C. Tong, L. J. Huang and J. L. Tan, Adv. Sci., 2018, 5, 1700611.

71 Q. J. Lv, L. L. Cheng, Y. Lu, X. G. Zhang, Y. Z. Wang, J. F. Deng, J. B. Zhou, B. Liu and J. Liu, Adv. Sci., 2020, 7, 2000515.

72 S.-H. Kwon, H. Al Faruque, H. Kee, E. Kim and S. Park, Colloids Surf., B, 2021, 205, 111915.

73 L. Cheng, X. Zhang, J. Tang, Q. Lv and J. Liu, Biomaterials, 2021, 275, 120964.

74 L. Sun, M. Fan, D. Huang, B. Li, R. Xu, F. Gao and Y. Chen, Biomaterials, 2021, 271, 120761.

75 Y. Hu, X. Li, Q. Zhang, Z. Gu, Y. Luo, J. Guo, X. Wang, Y. Jing, X. Chen and J. Su, Bioact. Mater., 2021, 6, 2905-2913.

76 G. Cheng, W. Q. Li, L. Ha, X. H. Han, S. J. Hao, Y. Wan, Z. G. Wang, F. P. Dong, X. Zou, Y. W. Mao and S. Y. Zheng, J. Am. Chem. Soc., 2018, 140, 7282-7291.

77 B. Illes, P. Hirschle, S. Baenert, V. Cauda, S. Wuttke and H. Engelke, Chem. Mater., 2017, 29, 8042-8046.

78 T. Y. Yong, X. Q. Zhang, N. N. Bie, H. B. Zhang, X. T. Zhang, F. Y. Li, A. Hakeem, J. Hu, L. Gan, H. A. Santos and X. L. Yang, Nat. Commun., 2019, 10, 3838.

79 Q. Liu, T. J. Fan, Y. Y. Zheng, S. L. Yang, Z. Q. Yu, Y. H. Duo, Y. H. Zhang, D. Adah, L. L. Shi, Z. Sun, D. Wang, J. L. Xie, H. Wu, Z. Z. Wu, C. C. Ge, L. J. Qiao, C. Y. Wei, L. D. Huang, Q. T. Yan, Q. H. Yang, S. Y. Bao, L. P. Liu and H. Zhang, Nanoscale, 2020, 12, 19939-19952.

80 D. Zhu, Z. Liu, Y. Li, Q. Huang, L. Xia and K. Li, Biomaterials, 2021, 274, 120894.

81 S. Y. Liu, X. Chen, L. L. Bao, T. Liu, P. Y. Yuan, X. S. Yang, X. Y. Qiu, J. J. Gooding, Y. K. Bai, J. J. Xiao, F. X. Pu and Y. Jin, Nat. Biomed. Eng., 2020, 4, 1063-1064.

82 S. Pan, Y. Zhang, M. Huang, Z. Deng, A. Zhang, L. Pei, L. Wang, W. Zhao, L. Ma, Q. Zhang and D. Cui, Biomaterials, 2021, 275, 120946.

83 M. Khongkow, T. Yata, S. Boonrungsiman, U. R. Ruktanonchai, D. Graham and K. Namdeel, Sci. Rep., 2019, 9, 8278. 
84 M. Sancho-Albero, N. Navascues, G. Mendoza, V. Sebastian, M. Arruebo, P. Martin-Duque and J. Santamaria, J. Nanobiotechnol., 2019, 17, 16.

85 M. Sancho-Albero, M. D. Encabo-Berzosa, M. BeltranVisiedo, L. Fernandez-Messina, V. Sebastian, F. SanchezMadrid, M. Arruebo, J. Santamaria and P. Martin-Duque, Nanoscale, 2019, 11, 18825-18836.

86 E. S. Lee, B. S. Cha, S. Kim and K. S. Park, Int. J. Mol. Sci., 2021, 22, 4433.

87 G. H. Nam, Y. Choi, G. B. Kim, S. Kim, S. A. Kim and I. S. Kim, Adv. Mater., 2020, 32, e2002440.

88 M. S. Yoon, Y. J. Lee, H. J. Shin, C. W. Park, S. B. Han, J. K. Jung, J. S. Kim and D. H. Shin, Pharmaceutics, 2020, 12, 1156.

89 L. Sercombe, T. Veerati, F. Moheimani, S. Y. Wu, A. K. Sood and S. Hua, Front. Pharmacol., 2015, 6, 286.

90 K. B. Knudsen, H. Northeved, P. E. K. Kumar, A. Permin, T. Gjetting, T. L. Andresen, S. Larsen, K. M. Wegener, J. Lykkesfeldt, K. Jantzen, S. Loft, P. Moller and M. Roursgaard, Nanomed. Nanotechnol., 2015, 11, 467-477.

91 H. Yang, Q. V. Le, G. Shim, Y. K. Oh and Y. K. Shin, Acta Pharm. Sin. B, 2020, 10, 2212-2226.

92 C. G. Yan, Y. K. Wu, J. Feng, W. F. Chen, X. Liu, P. Hao, R. M. Yang, J. Zhang, B. Q. Lin, Y. K. Xu and R. Y. Liu, Int. J. Nanomed., 2013, 8, 245-255.

93 D. S. Dimitrov, Y. Feng and P. Prabakaran, J. Comput. Theor. Nanosci., 2008, 5, 751-759.

94 L. Cheng, Q. S. Zhang, M. Xu, Q. C. Zhai and C. L. Zhang, J. Colloid Interface Sci., 2021, 583, 299-309.

95 W. Q. Wang, L. Wang, S. Liu and Z. G. Xie, Bioconjugate Chem., 2017, 28, 2784-2793.

96 Y. Cao, Y. Wang, X. M. Yu, X. H. Jiang, G. Li and J. Zhao, Biosens. Bioelectron., 2020, 166, 112452.

97 Y. T. Fu and Q. Ma, Nanoscale, 2020, 12, 13879-13898.

98 Y. Wang, Curr. Gene Ther., 2020, 20, 356-372.

99 E. J. Bunggulawa, W. Wang, T. Y. Yin, N. Wang, C. Durkan, Y. Z. Wang and G. X. Wang, J. Nanobiotechnol., 2018, 16, 81.

100 E. van der Pol, A. N. Boing, P. Harrison, A. Sturk and R. Nieuwland, Pharmacol. Rev., 2012, 64, 676-705.

101 S. El Andaloussi, I. Maeger, X. O. Breakefield and M. J. A. Wood, Nat. Rev. Drug Discovery, 2013, 12, 348-358.

102 M. Yang, X. Y. Liu, Q. Q. Luo, L. L. Xu and F. X. Chen, J. Nanobiotechnol., 2020, 18, 100.
103 E. Koh, E. J. Lee, G. H. Nam, Y. Hong, E. Cho, Y. Yang and I. S. Kim, Biomaterials, 2017, 121, 121-129.

104 E. Giacomini, S. Makieva, V. Murdica, R. Vago and P. Vigano, Curr. Opin. Obstet. Gyn., 2020, 32, 179-184.

105 K. O. Jung, Y. H. Kim, S. J. Chung, C. H. Lee, S. Rhee, G. Pratx, J. K. Chung and H. Youn, Int. J. Mol. Sci., 2020, 21, 7850 .

106 H. M. Saleh, F. A. El-Saied, T. A. Salaheldin and A. A. Hezo, Constr. Build. Mater., 2019, 218, 556-567.

107 P. Singh, S. Srivastava and S. K. Singh, ACS Biomater. Sci. Eng., 2019, 5, 4882-4898.

108 A. Tamayo, M. A. Mazo, R. Ruiz-Caro, A. Martin-Illana, L. M. Bedoya, M. D. Veiga-Ochoa and J. Rubio, Chem. Eng. J., 2015, 280, 165-174.

109 K. K. Qian and R. H. Bogner, J. Pharm. Sci., 2012, 101, 444-463.

110 L. Walekar, T. Dutta, P. Kumar, Y. S. Ok, S. Pawar, A. Deep and K. H. Kim, TrAC, Trends Anal. Chem., 2017, 97, 458-467.

111 P. Reineck, A. Francis, A. Orth, D. W. M. Lau, R. D. V. Nixon-Luke, I. Das Rastogi, W. A. W. Razali, N. M. Cordina, L. M. Parker, V. K. A. Sreenivasan, L. J. Brown and B. C. Gibson, Adv. Opt. Mater., 2016, 4, 1549-1557.

112 M. L. Lu, C. W. Lai, H. J. Pan, C. T. Chen, P. T. Chou and Y. F. Chen, Nano Lett., 2013, 13, 1920-1927.

113 M. Lannoo, C. Delerue, G. Allan and Y. M. Niquet, Philos. Trans. R. Soc., A, 2003, 361, 259-272.

114 F. Y. Song, P. S. Tang, H. Durst, D. T. Cramb and W. C. W. Chan, Angew. Chem., Int. Ed., 2012, 51, 8773-8777.

115 X. S. Tang, J. Yang, S. Q. Li, Z. Z. Liu, Z. P. Hu, J. Y. Hao, J. Du, Y. X. Leng, H. Y. Qin, X. Lin, Y. Lin, Y. X. Tian, M. Zhou and Q. H. Xiong, Adv. Sci., 2019, 6, 1900412.

116 G. Palui, S. Ray and A. Banerjee, J. Mater. Chem., 2009, 19, 3457-3468.

117 R. E. Messersmith, G. J. Nusz and S. M. Reed, J. Phys. Chem. C, 2013, 117, 26725-26733.

118 W. B. Ewe, H. S. Chu and E. P. Li, Opt. Express, 2007, 15, 18200-18208.

119 R. Rodriguez-Oliveros and J. A. Sanchez-Gil, Opt. Express, 2011, 19, 12208-12219. 